\title{
AN ANALYSIS OF THE MULTIPLICITY SPACES IN BRANCHING OF SYMPLECTIC GROUPS
}

\author{
ODED YACOBI
}

\begin{abstract}
Branching of symplectic groups is not multiplicity-free. We describe a new approach to resolving these multiplicities that is based on studying the associated branching algebra $\mathcal{B}$. The algebra $\mathcal{B}$ is a graded algebra whose components encode the multiplicities of irreducible representations of $\mathrm{Sp}_{2 n-2}$ in irreducible representations of $S p_{2 n}$. Our first theorem states that the map taking an element of $S p_{2 n}$ to its principal $n \times(n+1)$ submatrix induces an isomorphism of $\mathcal{B}$ to a different branching algebra $\mathcal{B}^{\prime}$. The algebra $\mathcal{B}^{\prime}$ encodes multiplicities of irreducible representations of $\mathrm{GL}_{n-1}$ in certain irreducible representations of $\mathrm{GL}_{n+1}$. Our second theorem is that each multiplicity space that arises in the restriction of an irreducible representation of $S p_{2 n}$ to $S p_{2 n-2}$ is canonically an irreducible module for the $n$-fold product of $S L_{2}$. In particular, this induces a canonical decomposition of the multiplicity spaces into one dimensional spaces, thereby resolving the multiplicities.
\end{abstract}

\section{CONTENTS}

1. Introduction

2. Preliminaries

2.1. Branching algebras

2.2. Notation

3. Main Results

3.1. An isomorphism of branching algebras

3.2. A resolution of multiplicities

4. Proof of Theorem 3.1

4.1. Some results of Zhelobenko

4.2. Preparatory lemmas

4.3. Proof of Theorem 3.1

5. Proof of Proposition 3.6

5.1. The rearrangement function

5.2. Proof of Proposition 3.2

5.3. A technical lemma

5.4. Proof of Proposition 3.6

6. Proof of Theorem 3.5

6.1. A filtration on the branching semigroup

6.2. Proof of Proposition 3.7

6.3. Proof of Theorem 3.5

2000 Mathematics Subject Classification. 20G05, 05E10.

Key words and phrases. symplectic group, branching algebra. 
7. Proof of Corollary $3.8 \quad 30$

References

\section{INTRODUCTION}

The purpose of this paper is to give a new interpretation of symplectic branching which, unlike the branching for the other towers of classical groups, is not multiplicity free. In other words, an irreducible representation of $S p_{2 n}$ does not decompose uniquely into irreducible representations of $\mathrm{Sp}_{2 n-2}$ (embedded as the subgroup fixing pointwise a two-dimensional non-isotropic subspace). We resolve this ambiguity by analyzing the algebraic structure of the associated multiplicity spaces.

Our main object of study is an algebra $\mathcal{B}$ associated to the pair $\left(S p_{2 n-2}, S p_{2 n}\right)$. This "branching algebra" is a graded algebra whose components are all the multiplicity spaces that appear in the restriction of irreducible representations of $S p_{2 n}$ to $S p_{2 n-2}$. By definition $\mathcal{B}$ is a certain subalgebra of the ring of regular functions, $\mathcal{O}\left(S p_{2 n}\right)$, on $S p_{2 n}$ :

$$
\mathcal{B}=\mathcal{O}\left(\overline{\mathrm{U}}_{\mathrm{C}_{n}} \backslash S p_{2 n} / \mathrm{U}_{\mathrm{C}_{n-1}}\right) .
$$

(See Section 2.2 for notation.) From this realization it follows that $\mathcal{B}$ has a natural action of $\mathrm{SL}_{2}$ by right translation.

Our first result relates $\mathcal{B}$ to a different branching algebra associated to the branching pair $\left(\mathrm{GL}_{n-1}, \mathrm{GL}_{n+1}\right)$. Using $\left(\mathrm{GL}_{n-1}, \mathrm{GL}_{n+1}\right)$-duality we define the algebra

$$
\mathcal{B}^{\prime}=\mathcal{O}\left(\overline{\mathrm{U}}_{\mathrm{n}} \backslash M_{\mathrm{n}, \mathrm{n}+1} / \mathrm{U}_{\mathrm{n}-1}\right) .
$$

This algebra is a "restricted" branching algebra, as it is isomorphic to a direct sum of only certain multiplicity spaces that occur in branching from $G L_{n+1}$ to $G L_{n-1}$. Note that $\mathcal{B}^{\prime}$ is also a graded $S L_{2}$-algebra. We consider the function $\psi: S p_{2 n} \rightarrow M_{n, n+1}$ which maps an element of $S p_{2 n}$ to its $n \times(n+1)$ principal submatrix. The theorem is that the induced map on functions $\psi^{*}: \mathcal{O}\left(M_{n, n+1}\right) \rightarrow \mathcal{O}\left(S p_{2 n}\right)$ restricts to give an isomorphism $\psi^{*}: \mathcal{B}^{\prime} \rightarrow \mathcal{B}$ of graded $\mathrm{SL}_{2}$-algebras.

This theorem allows us to reduce questions about branching from $S p_{2 n}$ to $S p_{2 n-2}$ to analogous questions concerning branching from $\mathrm{GL}_{n+1}$ to $\mathrm{GL}_{n-1}$. The latter are easier, as they can be "factored" through $\mathrm{GL}_{n}$. We will illustrate this reduction technique several times, most notably in order to prove our second theorem.

To describe our second theorem we introduce a family of subalgebras of $\mathcal{B}$ indexed by a finite set, $\Sigma$, of so-called order types. We prove that each subalgebra $\mathcal{B}_{\sigma}$ is isomorphic to the algebra, $\mathcal{O}(\mathrm{V})$, of polynomials on a vector space $\mathrm{V}$. This isomorphism is unique up to scalars. Moreover, $V$ can be given the structure of an $L=\prod_{i=1}^{n} S_{2}$-module. Therefore, via this isomorphism, we obtain a canonical action of $L$ on $\mathcal{B}_{\sigma}$ by algebra automorphisms. The action of $L$ is well-defined on the intersections of these subalgebras, allowing us to glue the modules together to obtain a representation, $\Phi$, of $L$ on $\mathcal{B}$.

The representation $(\Phi, \mathcal{B})$ of $L$ satisfies some remarkable properties. First and foremost, it identifies each multiplicity space as an explicit irreducible L-module. Secondly, the restriction of $\Phi$ to the diagonal subgroup of $L$ recovers the natural $S L_{2}$ action on 
$\mathcal{B}$. Finally, it is the unique such representation acting by algebra automorphisms on the subalgebras $\mathcal{B}_{\sigma}$.

As a corollary of this theorem we obtain resolution of the multiplicities that occur in branching of symplectic groups. Indeed, irreducible L-modules have one dimensional weight spaces. Therefore, via $\Phi$, we obtain a decomposition of the multiplicity spaces into one dimensional spaces. This decomposition is canonical, i.e. depends only the choice of torus of $\mathrm{Sp}_{2 \mathrm{n}}$ that is fixed throughout. The other known approach to this problem uses quantum groups, where these multiplicities are resolved using an infinite dimensional Hopf algebra called the twisted Yangian ([Mol99]).

The basis of $\mathcal{B}$ which we obtain from the action of $L$ is unique up to scalar. In [KY10] we study properties of this basis, and, in particular, show that it is a standard monomial basis, i.e. it satisfies a straightening algorithm. By induction this basis can be used to obtain a basis for irreducible representations of $S p_{2 n}$. The resulting basis is a partial analogue of the Gelfand-Zetlin basis ([GZ50]); to be properly called a "Gelfand-Zetlin" basis one must also compute the action of Chevalley generators. The only such basis known is the Gelfand-Zetlin-Molev basis arising from the Yangian theory mentioned above ([Mol99]). In a future work we will compare the basis resulting from our work to the Gelfand-Zetlin-Molev basis.

Acknowledgement. The results herein are based on the author's UC San Diego PhD thesis (2009). The author is grateful to his advisor, Nolan Wallach, for his guidance and insights. The author also thanks Avraham Aizenbud, Sangjib Kim, Allen Knutson and Gerald Schwarz for helpful conversations. This work was supported in part by the ARCS Foundation.

\section{PRELIMinaries}

Our main object of study, $\mathcal{B}$, is an example of a branching algebra. In section 2.1 we define branching algebras and their associated branching semigroups. In section 2.2 we fix some notation that will be used throughout.

2.1. Branching algebras. Let $G$ be a connected classical group with identity $e \in G$. Fix a maximal torus $T_{G}$, Borel subgroup $B_{G}$, and unipotent radical $U_{G} \subset B_{G}$ so that $\mathrm{T}_{\mathrm{G}} \mathrm{U}_{\mathrm{G}}=\mathrm{B}_{\mathrm{G}}$. Let $\overline{\mathrm{U}}_{\mathrm{G}}$ be the unipotent group opposite $\mathrm{U}_{\mathrm{G}}$. When convenient, we work in the setting of Lie algebras. We denote the complex Lie algebra of a complex Lie group by the corresponding lower-case fraktur letter.

Fix the choice of positive roots giving $\mathrm{U}_{\mathrm{G}}: \Phi_{\mathrm{G}}^{+}=\Phi\left(\mathfrak{b}_{\mathrm{G}}, \mathfrak{t}_{\mathrm{G}}\right)$. Let $\wedge_{\mathrm{G}} \subset \mathfrak{t}^{*}$ be the corresponding semigroup of dominant integral weights. Denote by $F_{G}^{\lambda}$ the finite-dimensional irreducible representation of $G$ of highest weight $\lambda \in \Lambda_{G}$.

For an affine algebraic variety $X$, let $\mathcal{O}(X)$ denote the algebra of regular functions on $X$. The group $G$ has the structure of an affine algebraic variety, and $\mathcal{O}(\mathrm{G})$ is a $\mathrm{G} \times \mathrm{G}$-module under left and right translation. Let $\mathcal{R}_{\mathrm{G}}=\mathcal{O}\left(\overline{\mathrm{U}}_{\mathrm{G}} \backslash \mathrm{G}\right)$ be the left $\overline{\mathrm{U}}_{\mathrm{G}}$-invariant functions on $\mathrm{G}$ :

$$
\mathcal{R}_{\mathrm{G}}=\left\{f \in \mathcal{O}(\mathrm{G}): \mathrm{f}(\overline{\mathrm{u}} \mathrm{g})=\mathrm{f}(\mathrm{g}) \text { for all } \overline{\mathrm{u}} \in \overline{\mathrm{U}}_{\mathrm{G}} \text { and } \mathrm{g} \in \mathrm{G}\right\} \text {. }
$$


Under the right action of G (see Theorem 4.2.7. , GW09]):

$$
\mathcal{R}_{\mathrm{G}} \cong \bigoplus_{\lambda \in \Lambda_{\mathrm{G}}}\left(\left(\mathrm{F}_{\mathrm{G}}^{\lambda}\right)^{*}\right)^{\overline{\mathrm{u}}_{\mathrm{G}}} \otimes \mathrm{F}_{\mathrm{G}}^{\lambda} \cong \bigoplus_{\lambda \in \Lambda_{\mathrm{G}}} \mathrm{F}_{\mathrm{G}}^{\lambda} .
$$

Henceforth identify $F_{G}^{\lambda}$ with its image in $\mathcal{R}_{G}$. Let $f_{G}^{\lambda} \in F_{G}^{\lambda}$ be the unique highest weight vector such that $f_{G}^{\lambda}(e)=1$. We call $f_{G}^{\lambda}$ the canonical highest weight vector of $F_{G}^{\lambda}$. Now let $\lambda, \lambda^{\prime} \in \Lambda_{G}$. Then $f_{G}^{\lambda} f_{G}^{\lambda^{\prime}}$ is $U$-invariant of weight $\lambda+\lambda^{\prime}$. Since also $f_{G}^{\lambda} f_{G}^{\lambda^{\prime}}(e)=1$, it follows that $f_{G}^{\lambda} f_{G}^{\lambda^{\prime}}=f_{G}^{\lambda+\lambda^{\prime}}$, and therefore $F_{G}^{\lambda} F_{G}^{\lambda^{\prime}}=F_{G}^{\lambda+\lambda^{\prime}}$.

The Cartan product, $\pi_{\lambda, \lambda^{\prime}}: \mathrm{F}_{\mathrm{G}}^{\lambda} \otimes \mathrm{F}_{\mathrm{G}}^{\lambda^{\prime}} \rightarrow \mathrm{F}_{\mathrm{G}}^{\lambda+\lambda^{\prime}}$, is defined by $\pi_{\lambda, \lambda^{\prime}}\left(v \otimes v^{\prime}\right)=v v^{\prime}$. The Cartan embedding, $j_{\lambda, \lambda^{\prime}}: F_{G}^{\lambda+\lambda^{\prime}} \rightarrow F_{G}^{\lambda} \otimes F_{G}^{\lambda^{\prime}}$, is given by setting $j_{\lambda, \lambda^{\prime}}\left(f_{G}^{\lambda+\lambda^{\prime}}\right)=f_{G}^{\lambda} \otimes f_{G}^{\lambda^{\prime}}$, and extending by G-linearity.

Suppose now that $\mathrm{H} \subset \mathrm{G}$ is a connected Lie subgroup, and we have chosen its distinguished subgroups so that $\overline{\mathrm{U}}_{\mathrm{H}} \subset \overline{\mathrm{U}}_{\mathrm{G}}, \mathrm{T}_{\mathrm{H}} \subset \mathrm{T}_{\mathrm{G}}$, and $\mathrm{U}_{\mathrm{H}} \subset \mathrm{U}_{\mathrm{G}}$. Consider the subalgebra of bi-invariants

$$
\mathcal{B}(\mathrm{H}, \mathrm{G})=\mathcal{O}\left(\overline{\mathrm{U}}_{\mathrm{G}} \backslash \mathrm{G} / \mathrm{U}_{\mathrm{H}}\right) \subset \mathcal{R}_{\mathrm{G}} .
$$

In other words, $\mathcal{B}(\mathrm{H}, \mathrm{G})$ consists of the functions $f \in \mathcal{R}_{\mathrm{G}}$ such that $\mathrm{f}(\mathrm{gu})=\mathrm{f}(\mathrm{g})$ for all $\mathrm{g} \in \mathrm{G}$ and $\mathrm{u} \in \mathrm{U}_{\mathrm{H}}$.

Since $T_{H}$ normalizes $U_{H}$ there is an action of $T_{H}$ on $\mathcal{B}(H, G)$ by right translation. The decomposition of $\mathcal{B}(\mathrm{H}, \mathrm{G})$ into $\mathrm{T}_{\mathrm{H}}$ weight spaces is:

$$
\mathcal{B}(\mathrm{H}, \mathrm{G})=\bigoplus_{(\mu, \lambda) \in \Lambda_{\mathrm{H}} \times \Lambda_{\mathrm{G}}} \mathrm{F}^{\lambda / \mu}
$$

The weight space $\mathrm{F}^{\lambda / \mu}$ is defined as

$$
F^{\lambda / \mu}=\left\{f \in\left(F_{G}^{\lambda}\right)^{U_{H}}: f(g t)=t^{\mu} f(g) \text { for all } g \in G \text { and } t \in T_{H}\right\} .
$$

Equivalently, $F^{\lambda / \mu}$ is the $T_{G} \times T_{H}$ weight space of $\mathcal{B}$ corresponding to the weight $(-\lambda)$ for $\mathrm{T}_{\mathrm{G}}$ and $\mu$ for $\mathrm{T}_{\mathrm{H}}$.

Note that the centralizer $\mathrm{Z}_{\mathrm{G}}(\mathrm{H})$ acts on $\mathrm{F}^{\lambda / \mu}$ by right translation. As a $\mathrm{Z}_{\mathrm{G}}(\mathrm{H})$-module there is a canonical isomorphism

$$
\mathrm{F}^{\lambda / \mu} \cong \operatorname{Hom}_{H}\left(\mathrm{~F}_{\mathrm{H}}^{\mu}, \mathrm{F}_{\mathrm{G}}^{\lambda}\right) .
$$

(The isomorphism maps $\phi \in \operatorname{Hom}_{H}\left(\mathrm{~F}_{\mathrm{H}}^{\mu}, \mathrm{F}_{\mathrm{G}}^{\lambda}\right)$ to $\phi\left(\mathrm{f}_{\mathrm{H}}^{\mu}\right) \in \mathrm{F}_{\lambda / \mu}$.) In particular, the dimension of $F^{\lambda / \mu}$ counts the branching multiplicity of $F_{H}^{\mu}$ in the restriction to $H$ of $F_{G}^{\lambda}$. For this reason $\mathcal{B}(\mathrm{H}, \mathrm{G})$ is termed the branching algebra for the pair $(\mathrm{H}, \mathrm{G})$ (cf. [HTW08], [Zh73] and references therein).

Lemma 2.1. Let $(\mu, \lambda),\left(\mu^{\prime}, \lambda^{\prime}\right) \in \Lambda_{H} \times \Lambda_{G}$. Then

$$
\pi_{\lambda, \lambda^{\prime}}\left(F^{\lambda / \mu} \otimes F^{\lambda^{\prime} / \mu^{\prime}}\right) \subset F^{\lambda+\lambda^{\prime} / \mu+\mu^{\prime}} .
$$

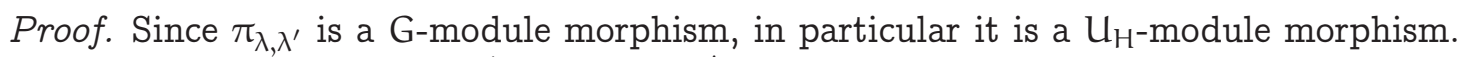
Therefore, $\pi_{\lambda, \lambda^{\prime}}\left(\left(F_{G}^{\lambda}\right)^{\mathrm{u}_{H}} \otimes\left(F_{G}^{\lambda^{\prime}}\right)^{\mathrm{U}_{H}}\right) \subset\left(F_{G}^{\lambda+\lambda^{\prime}}\right)^{\mathrm{u}_{H}}$. Now note that $\pi_{\lambda, \lambda^{\prime}}$ intertwines the $T_{H}$ action on $\left(F_{G}^{\lambda}\right)^{U_{H}} \otimes\left(F^{\lambda^{\prime}}\right)_{G}^{U_{H}}$ and $\left(F_{G}^{\lambda+\lambda^{\prime}}\right)^{U_{H}}$. Let $\left.\left(\left(F_{G}^{\lambda}\right)^{U_{H}} \otimes\left(F_{G}^{\lambda^{\prime}}\right)^{U_{H}}\right)\right)\left(\mu+\mu^{\prime}\right)$ be the $\mu+\mu^{\prime}$ weight space of $T_{H}$ on $\left.\left(F_{G}^{\lambda}\right)^{u_{H}} \otimes\left(F_{G}^{\lambda^{\prime}}\right)^{u_{H}}\right)$. Then $\pi_{\lambda, \lambda^{\prime}}$ maps $\left.\left(\left(F_{G}^{\lambda}\right)^{U_{H}} \otimes\left(F_{G}^{\lambda^{\prime}}\right)^{u_{H}}\right)\right)\left(\mu+\mu^{\prime}\right)$ to 
the weight space $F^{\lambda+\lambda^{\prime} / \mu+\mu^{\prime}}$. Since $F^{\lambda / \mu} \otimes F^{\lambda^{\prime} / \mu^{\prime}} \subset\left(\left(F_{G}^{\lambda}\right)^{U_{H}} \otimes\left(F_{G}^{\lambda^{\prime}}\right)^{U_{H}}\right)\left(\mu+\mu^{\prime}\right)$ the proof is complete.

By the lemma $\mathcal{B}(H, G)$ is a $\Lambda_{H} \times \Lambda_{G}$-graded algebra. Abusing notation a bit, we denote the restriction of $\pi_{\lambda, \lambda^{\prime}}$ to $F^{\lambda / \mu} \otimes F^{\lambda^{\prime} / \mu^{\prime}}$ also by $\pi_{\lambda, \lambda^{\prime}}$. This will cause no confusion since we will explicitly write

$$
F^{\lambda / \mu} \otimes F^{\lambda^{\prime} / \mu^{\prime}} \stackrel{\pi_{\lambda, \lambda^{\prime}}}{\longrightarrow} F^{\lambda+\lambda^{\prime} / \mu+\mu^{\prime}}
$$

when referring to this map, which we call the Cartan product of multiplicity spaces. In general the Cartan product of multiplicity spaces is not surjective (see section Proposition 3.6). This observation will be critical.

We can associate to the pair $(H, G)$ the following set:

$$
\Lambda_{\mathcal{B}(H, G)}=\left\{(\mu, \lambda) \in \Lambda_{H} \times \Lambda_{G}: F^{\lambda / \mu} \neq\{0\}\right\} .
$$

Lemma 2.2. The set $\Lambda_{\mathcal{B}(\mathrm{H}, \mathrm{G})}$ is a semigroup under entry-wise addition.

Proof. Suppose $(\mu, \lambda),\left(\mu^{\prime}, \lambda^{\prime}\right) \in \Lambda_{\mathcal{B}(\mathrm{H}, \mathrm{G})}$. Choose $0 \neq x \in \mathrm{F}^{\lambda / \mu}$ and $0 \neq x^{\prime} \in \mathrm{F}^{\lambda^{\prime} / \mu^{\prime}}$. Since $\mathcal{B}(H, G)$ has no zero divisors, $x x^{\prime} \neq 0$. By the above lemma this implies that $\mathrm{F}^{\lambda+\lambda^{\prime} / \mu+\mu^{\prime}} \neq 0$, i.e. $\left(\mu+\mu^{\prime}, \lambda+\lambda^{\prime}\right) \in \Lambda_{\mathcal{B}(\mathrm{H}, \mathrm{G})}$. The other semigroup axioms are trivial to check.

Thus the $\Lambda_{H} \times \Lambda_{G}$-graded algebra $\mathcal{B}(H, G)$ can also be regarded as a $\Lambda_{\mathcal{B}(H, G) \text {-graded }}$ algebra, and we call $\Lambda_{\mathcal{B}(\mathrm{H}, \mathrm{G})}$ a branching semigroup.

2.2. Notation. Henceforth fix an integer $n>1$. Let $\Lambda_{n}$ be the semigroup of weakly decreasing sequences of length $n$ consisting of non-negative integers. For $m \geq 1$, let $\Lambda_{\mathrm{m}, \mathrm{n}}=\Lambda_{\mathrm{m}} \times \Lambda_{\mathrm{n}}$.

The set of dominant weights for irreducible polynomial representations of $\mathrm{GL}_{n}=$ $\mathrm{GL}(\mathrm{n}, \mathbb{C})$ is identified with $\Lambda_{n}$ in the usual way (see e.g. Theorem 5.5.22., [GW09]). For $\lambda \in \Lambda_{n}$, let $V^{\lambda}=F_{G L_{n}}^{\lambda}$ be the irreducible representation of $G L_{n}$ with highest weight $\lambda$, which we realize in $\mathcal{R}_{\mathrm{GL}}$ as described in Section 2.1. Let $v_{\lambda} \in \mathrm{V}^{\lambda}$ be the canonical highest weight vector.

Let $T_{n}$ be the subgroup of diagonal matrices in $G_{n}, U_{n}$ the subgroup of uppertriangular unipotent matrices, and $\overline{\mathrm{U}}_{n}$ be the subgroup of lower-triangular unipotent matrices. Suppose $1 \leq \mathrm{m}<\mathrm{n}$. We embed $\mathrm{GL}_{\mathrm{m}}$ in $\mathrm{GL}_{\mathrm{n}}$ as the subgroup:

$$
\left\{\left[\begin{array}{ll}
g & \\
& I_{n-m}
\end{array}\right]: g \in G_{m}\right\} \text {. }
$$

For $(\mu, \lambda) \in \Lambda_{m, n}$, define the multiplicity space $V^{\lambda / \mu}$ as in (1) with $H=G L_{m}$ and $\mathrm{G}=\mathrm{GL}_{n}$. In the case $\mathrm{m}=\mathrm{n}-1$ these spaces are classically known (see e.g. Theorem 8.1.1., [GW09]): for $(\mu, \lambda) \in \Lambda_{n-1, n}$,

$$
\begin{aligned}
\operatorname{dim} V^{\lambda / \mu} & \leq 1 \\
V^{\lambda / \mu} \neq\{0\} & \Leftrightarrow \mu \text { interlaces } \lambda .
\end{aligned}
$$

The interlacing condition, written $\mu<\lambda$, means that $\lambda_{i} \geq \mu_{i} \geq \lambda_{i+1}$ for $i=1, \ldots, n-1$, where $\mu=\left(\mu_{1}, \ldots, \mu_{n-1}\right)$ and $\lambda=\left(\lambda_{1}, \ldots, \lambda_{n}\right)$. 
Let $M_{m, n}$ denote the space of $m \times n$ matrices with complex entries. By $\left(G L_{n}, G L_{n+1}\right)$ duality, the space of polynomials $\mathcal{O}\left(M_{n, n+1}\right)$ decomposes multiplicity free as $G L_{n} \times G L_{n+1}$ module (see e.g. Theorem 5.6.7.,[GW09]):

$$
\mathcal{O}\left(M_{n, n+1}\right) \cong \bigoplus_{\lambda \in \Lambda_{n}} V^{\lambda^{*}} \otimes V^{\lambda^{+}}
$$

Here $\lambda^{+} \in \Lambda_{n+1}$ is obtained from $\lambda$ by adding a zero. Notice that $\mathrm{V}^{\lambda^{*}}$ is an irreducible representation of $G L_{n}$, while $V^{\lambda^{+}}$is an irreducible representation of $G L_{n+1}$.

We will study the algebra of bi-invariants

$$
\mathcal{B}^{\prime}=\mathcal{O}\left(\overline{\mathrm{U}}_{\mathrm{n}} \backslash \mathrm{M}_{\mathrm{n}, \mathrm{n}+1} / \mathrm{U}_{\mathrm{n}-1}\right) \cong \bigoplus_{(\mu, \lambda) \in \Lambda_{\mathrm{n}-1, \mathrm{n}}} \mathrm{V}^{\lambda^{+} / \mu}
$$

This algebra is a sort of "restricted" branching algebra as it includes only certain multiplicity spaces that occur in branching from $\mathrm{GL}_{n+1}$ to $\mathrm{GL}_{n-1}$.

The algebra $\mathcal{B}^{\prime}$ is an $\Lambda_{n-1, n}$-graded algebra; the $(\mu, \lambda)$ component is $\mathrm{V}^{\lambda^{+} / \mu}$. Moreover, it is naturally an $\mathrm{SL}_{2}$-algebra. Indeed, there is an obvious $\mathrm{SL}_{2} \subset \mathrm{GL}_{n+1}$ that commutes with $\mathrm{GL}_{n-1}$, and therefore acts on $\mathcal{B}^{\prime}$ by right translation. This action clearly preserves the graded components of $\mathcal{B}^{\prime}$, i.e. the multiplicity spaces $\mathrm{V}^{\lambda^{+} / \mu}$ are naturally $\mathrm{SL}_{2}$-modules.

By Lemma 2.2, the algebra $\mathcal{B}^{\prime}$ is also graded by the semigroup

$$
\Lambda_{\mathcal{B}^{\prime}}=\left\{(\mu, \lambda) \in \Lambda_{n-1, n}: V^{\lambda^{+} / \mu} \neq\{0\}\right\} .
$$

It will be useful for us to have a more concrete realization of this semigroup. Suppose $\mu=\left(\mu_{1}, \ldots, \mu_{n-1}\right) \in \Lambda_{n-1}$ and $\lambda=\left(\lambda_{1}, \ldots, \lambda_{n+1}\right) \in \Lambda_{n+1}$. We say $\mu$ double interlaces $\lambda$, written $\mu \ll \lambda$, if for $i=1, \ldots, n-1$,

$$
\lambda_{i} \geq \mu_{i} \geq \lambda_{i+2} \text {. }
$$

If $(\mu, \lambda) \in \Lambda_{n-1, n}$, then $\mu$ double interlaces $\lambda$, also written $\mu \ll \lambda$, if $\mu \ll \lambda^{+}$.

Suppose $(\mu, \lambda) \in \Lambda_{n-1, n}$. It follows that $\mu \ll \lambda$ if, and only if, there exists $\gamma \in \Lambda_{n}$ such that $\mu<\gamma<\lambda^{+}$(see Lemma 5.1). Therefore by (3),

$$
\Lambda_{\mathcal{B}^{\prime}}=\left\{(\mu, \lambda) \in \Lambda_{n-1, n}: \mu \ll \lambda\right\} .
$$

Next we consider the symplectic groups. Label a basis for $\mathbb{C}^{2 n}$ as $e_{ \pm 1}, \ldots, e_{ \pm n}$ where $e_{-i}=e_{2 n+1-i}$. Denote by $s_{n}$ the $n \times n$ matrix with one's on the anti-diagonal and zeros everywhere else. Set

$$
J_{n}=\left[\begin{array}{cc}
0 & s_{n} \\
-s_{n} & 0
\end{array}\right]
$$

and consider the skew-symmetric bilinear form $\Omega_{n}(x, y)=x^{t} J_{n} y$ on $\mathbb{C}^{2 n}$. Define the symplectic group relative to this form: $S p_{2 n}=S p\left(\mathbb{C}^{2 n}, \Omega\right)$. In this realization we can take as a maximal torus $T_{C_{n}}=T_{2 n} \cap S p_{2 n}$, a maximal unipotent subgroup $U_{C_{n}}=U_{2 n} \cap S p_{2 n}$, and its opposite $\overline{\mathrm{U}}_{\mathrm{C}_{\mathrm{n}}}=\overline{\mathrm{U}}_{2 \mathrm{n}} \cap \mathrm{S} \mathrm{p}_{2 \mathrm{n}}$

Embed $S p_{2 n-2}$ in $S p_{2 n}$ as the subgroup fixing the vectors $e_{ \pm n}$. Notice that

$$
\left\{g \in S p_{2 n}: g e_{ \pm i}=e_{ \pm i} \text { for } i=1, \ldots, n-1\right\}
$$


is a subgroup isomorphic to $\mathrm{SL}_{2}=\mathrm{Sp}_{2}$ commuting with $S \mathrm{p}_{2 \mathrm{n}-2}$.

The set of dominant weights for $S p_{2 n}$ is identified with $\Lambda_{n}$ (see e.g. Theorem 3.1.20., [GW09]). For $\lambda \in \Lambda_{n}$, let $W^{\lambda}=F_{S p_{2 n}}^{\lambda}$ be the irreducible representation of $S p_{2 n}$ with highest weight $\lambda$, which we realize in $\mathcal{R}_{S_{p_{2 n}}}$ as described in section 2.1. Let $w_{\lambda} \in W^{\lambda}$ be the canonical highest weight vector.

For $(\mu, \lambda) \in \Lambda_{n-1, n}$ we define the multiplicity space $W^{\lambda / \mu}$ as in (11) with $H=S p_{2 n-2}$ and $G=S p_{2 n}$. Therefore the dimension of $W^{\lambda / \mu}$ is the multiplicity of the irreducible representation $W^{\mu}$ of $S p_{2 n-2}$ in the representation $W^{\lambda}$ of $S p_{2 n}$. In contrast to the general linear groups, the branching of the symplectic groups is not multiplicity-free, i.e. $\operatorname{dim} W^{\lambda / \mu}>1$ for generic $(\mu, \lambda) \in \Lambda_{n-1, n}$ (see Corollary 3.3).

Our main object of study is the following branching algebra:

$$
\mathcal{B}=\mathcal{B}\left(S p_{2 n-2}, S p_{2 n}\right) .
$$

By Lemma 2.1, $\mathcal{B}$ is graded by $\wedge_{n-1, n}$ :

$$
\mathcal{B}=\bigoplus_{(\mu, \lambda) \in \Lambda_{n-1, n}} W^{\lambda / \mu}
$$

By Lemma 2.2, we also consider $\mathcal{B}$ as graded over the branching semigroup

$$
\Lambda_{\mathcal{B}}=\Lambda\left(S p_{2 n-2}, S p_{2 n}\right) .
$$

The branching algebra $\mathcal{B}$ is naturally an $\mathrm{SL}_{2}$-module for the copy of $\mathrm{SL}_{2}$ appearing in (6). Indeed, this copy $S_{2}$ acts on $\mathcal{B}$ by right translation leaving the graded components $W^{\lambda / \mu}$ invariant. In other words, the multiplicity spaces $W^{\lambda / \mu}$ are naturally $S L_{2}$-modules. We refer to this action as the "natural" $S L_{2}$ action, and denote it simply by $x . b$ for $x \in \mathrm{SL}_{2}$ and $\mathrm{b} \in \mathcal{B}$.

Finally, we consider the group $\mathrm{SL}_{2}=\operatorname{SL}(2, \mathbb{C})$. Let $\mathrm{F}^{\mathrm{k}}=\mathcal{O}^{\mathrm{k}}\left(\mathbb{C}^{2}\right)$ be the $(\mathrm{k}+1)^{\mathrm{th}}$ dimensional irreducible representation of $\mathrm{SL}_{2}$, realized as the polynomials on $\mathbb{C}^{2}$ of homogeneous degree $k$. The $\mathrm{SL}_{2}$ action is by right translation. For $k, k^{\prime} \geq 0$ let $\pi_{k, k^{\prime}}: F^{k} \otimes F^{k^{\prime}} \rightarrow F^{k+k^{\prime}}$ be the usual multiplication of functions, and define the embedding of $\mathrm{SL}_{2}$-modules, $j_{k, k^{\prime}}: F^{k+k^{\prime}} \rightarrow F^{k} \otimes F^{k^{\prime}}$, by setting $j_{k, k^{\prime}}\left(x_{1}^{k+k^{\prime}}\right)=x_{1}^{k} \otimes x_{1}^{k^{\prime}}$ and extending by $S L_{2}$-linearity. Here $x_{1}$ is the first coordinate function on $\mathbb{C}^{2}$.

Remark 2.3. We use the symbols $\pi_{\lambda, \lambda^{\prime}}$ and $j_{\lambda, \lambda^{\prime}}$ to denote the Cartan maps for any of the given groups above. It will be clear from context which group we have in mind.

\section{Main Results}

In this section we describe in detail the two main theorems of this paper.

3.1. An isomorphism of branching algebras. Our first theorem describes an isomorphism of the branching algebras $\mathcal{B}$ and $\mathcal{B}^{\prime}$. This usefulness of this theorem will become clear, as we use it repeatedly to reduce questions about branching of the symplectic groups to analogous questions about branching of the general linear groups. 
Define the map $\psi: S p_{2 n} \rightarrow M_{n, n+1}$, which assigns an element $g \in S p_{2 n}$ its principal $n \times(n+1)$ submatrix. Consider the induced map on functions

$$
\psi^{*}: \mathcal{O}\left(M_{n, n+1}\right) \rightarrow \mathcal{O}\left(S_{2 n}\right) .
$$

In Lemma 4.6 we show that $\psi^{*}\left(\mathcal{B}^{\prime}\right) \subset \mathcal{B}$, and, moreover, that $\psi^{*}: \mathcal{B}^{\prime} \rightarrow \mathcal{B}$ is a map of $\Lambda_{n-1, n}$-graded, $S L_{2}$ algebras. In fact, much more is true:

Theorem 3.1. The map $\psi^{*}: \mathcal{B}^{\prime} \rightarrow \mathcal{B}$ is an isomorphism of $\Lambda_{n-1, n}$-graded, $\mathrm{SL}_{2}-$ algebras.

We now describe some applications.

For our first application let $(\mu, \lambda) \in \Lambda_{n-1, n}$. By the theorem,

$$
W^{\lambda / \mu} \neq\{0\} \Leftrightarrow V^{\lambda^{+} / \mu} \neq\{0\} .
$$

Combining this with (5), we recover a classical result about symplectic branching (see e.g. Theorem 8.1.5., [GW09]):

$$
W^{\lambda / \mu} \neq\{0\} \Leftrightarrow \mu \ll \lambda,
$$

i.e. $\Lambda_{\mathcal{B}}=\Lambda_{\mathcal{B}^{\prime}}$.

To describe our second application of this theorem, recall first that the multiplicity spaces $W^{\lambda / \mu}$ are each $S_{2}$-modules. Naturally, one would like to describe the $\mathrm{SL}_{2}$-module structure of these multiplicity spaces. By Theorem 3.1, $W^{\lambda / \mu} \cong V^{\lambda^{+} / \mu}$ as $\mathrm{SL}_{2}$-modules, so it suffices to answer the analogous question for the general linear groups. But this is not too difficult, since branching from $G L_{n+1}$ to $G L_{n-1}$ factors through $G L_{n}$.

Given $(\mu, \lambda) \in \Lambda_{n-1, n+1}$ let $\left(x_{1} \geq y_{1} \geq \cdots \geq x_{n} \geq y_{n}\right)$ be the non-increasing rearrangement of $\left(\mu_{1}, \ldots, \mu_{n-1}, \lambda_{1}, \ldots, \lambda_{n+1}\right)$. Set

$$
r_{i}(\mu, \lambda)=x_{i}-y_{i} .
$$

Proposition 3.2. Suppose $(\mu, \lambda) \in \Lambda_{n-1, n+1}$, and $\mu \ll \lambda$. Then as $\mathrm{SL}_{2}$-modules

$$
V^{\lambda / \mu} \cong \bigotimes_{i=1}^{n} F^{r_{i}(\mu, \lambda)}
$$

where $\mathrm{SL}_{2}$ acts by the tensor product representation on the right hand side.

As a corollary of Theorem 3.1 and Proposition 3.2 we can describe the $\mathrm{SL}_{2}$-module structure of the multiplicity spaces $W^{\lambda / \mu}$.

Corollary 3.3. Suppose $(\mu, \lambda) \in \Lambda_{\mathcal{B}}$. Then as $\mathrm{SL}_{2}$-modules

$$
W^{\lambda / \mu} \cong \bigotimes_{i=1}^{n} F^{r_{i}\left(\mu, \lambda^{+}\right)} \text {, }
$$

where $\mathrm{SL}_{2}$ acts by the tensor product representation on the right hand side.

The above corollary first appeared explicitly as Theorem 3.3, [WY], where it was proved using the combinatorics of partition functions. It can also be obtained using Theorem 5.2, Mol99, where it is shown that $W^{\lambda / \mu}$ carries an irreducible action of a certain infinite dimensional Hopf algebra called the twisted Yangian. 
3.2. A resolution of multiplicities. We now describe the second theorem of this paper. Define $L=\prod_{i=1}^{n} S_{2}$. For $(\mu, \lambda) \in \Lambda_{n-1, n}$ consider the irreducible L-module

$$
A^{\lambda / \mu}=\bigotimes_{i=1}^{n} F^{r_{i}\left(\mu, \lambda^{+}\right)} \text {. }
$$

Corollary 3.3 states that for all $(\mu, \lambda) \in \Lambda_{\mathcal{B}}, W^{\lambda / \mu} \cong \operatorname{Res}_{S L_{2}}^{L} A^{\lambda / \mu}$, where $S L_{2} \subset L$ is the diagonal subgroup. We therefore ask, is there a canonical action of $L$ on $W^{\lambda / \mu}$ such that $W^{\lambda / \mu} \cong A^{\lambda / \mu}$ as L-modules?

Remarkably, the answer to this question is yes! We will construct a canonical action of $\mathrm{L}$ on $\mathcal{B}$ that will be uniquely determined by two properties, the first of which is that each multiplicity space $W^{\lambda / \mu}$ is isomorphic to $A^{\lambda / \mu}$ as an L-module. Moreover, the restriction of this action to the diagonally embedded $\mathrm{SL}_{2} \subset \mathrm{L}$ recovers the natural action of $\mathrm{SL}_{2}$ on $\mathcal{B}$.

We warn the reader that $\mathrm{L}$ is not the product of $\mathrm{SL}_{2}$ 's that lives in $\mathrm{Sp}_{2 \mathrm{n}}$. Indeed, the latter product of $\mathrm{SL}_{2}$ 's does not act on the multiplicity spaces. The existence of this L-action is more subtle, and can only be "seen" by considering all multiplicity spaces together, i.e. by considering the branching algebra.

To describe the action of $\mathrm{L}$ on $\mathcal{B}$ precisely we investigate the double interlacing condition that characterizes branching of the symplectic groups. Notice that the inequality

$$
\lambda_{i} \geq \mu_{i} \geq \lambda_{i+2}
$$

does not constrain the relation between $\mu_{i}$ and $\lambda_{i+1}$. In other words, we can have either $\mu_{i} \geq \lambda_{i+1}$, or $\mu_{i} \leq \lambda_{i+1}$, or both. This motivates the following:

Definition 3.4. An order type $\sigma$ is a word in the alphabet $\{\geq, \leq\}$ of length $n-1$.

Suppose $(\mu, \lambda) \in \Lambda_{\mathcal{B}}$ and $\sigma=\left(\sigma_{1} \cdots \sigma_{n-1}\right)$ is an order type. Then we say $(\mu, \lambda)$ is of order type $\sigma$ if for $i=1, \ldots, n-1$,

$$
\left\{\begin{array}{l}
\sigma_{i}=" \geq " \Longrightarrow \mu_{i} \geq \lambda_{i+1} \\
\sigma_{i}=" \leq " \Longrightarrow \mu_{i} \leq \lambda_{i+1}
\end{array}\right.
$$

For example, consider the double interlacing pair $(\mu, \lambda)$, where $\lambda=(3,2,1)$ and $\mu=(3,0)$. Since $\mu_{1} \geq \lambda_{2}$ and $\mu_{2} \leq \lambda_{3}$, the pair $(\mu, \lambda)$ is of order type $\sigma=(\geq \leq)$.

Let $\Sigma$ be the set of order types, and for each $\sigma \in \Sigma$ set

$$
\Lambda_{\mathcal{B}}(\sigma)=\left\{(\mu, \lambda) \in \Lambda_{\mathcal{B}}:(\mu, \lambda) \text { is of order type } \sigma\right\} .
$$

It's easy to check that $\Lambda_{\mathcal{B}}(\sigma)$ is a sub-semigroup of $\Lambda_{\mathcal{B}}$. Therefore

$$
\mathcal{B}_{\sigma}=\bigoplus_{(\mu, \lambda) \in \Lambda_{\mathcal{B}}(\sigma)} W^{\lambda / \mu}
$$

is a subalgebra of $\mathcal{B}$. Moreover, $\mathcal{B}_{\sigma}$ is $\mathrm{SL}_{2}$-invariant. We now have all the ingredients to state our second theorem.

Theorem 3.5. There is a unique representation $(\Phi, \mathcal{B})$ of $\mathrm{L}$ satisfying the following two properties: 
(1) for all $(\mu, \lambda) \in \Lambda_{\mathcal{B}}, W^{\lambda / \mu}$ is an irreducible L-invariant subspace of $\mathcal{B}$ isomorphic to $A^{\lambda / \mu}$, and

(2) for all $\sigma \in \Sigma$, L acts as algebra automorphisms on $\mathcal{B}_{\sigma}$. Moreover, $\operatorname{Res}_{\mathrm{S}_{2}}^{\mathrm{L}}(\Phi)$ recovers the natural action of $\mathrm{SL}_{2}$ on $\mathcal{B}$.

We will now give an overview of the proof of this theorem. From the statement of Theorem 3.5 it's clear that the subalgebras $\mathcal{B}_{\sigma}$ are intrinsic to the definition of the representation $(\Phi, \mathcal{B})$ of $\mathrm{L}$. Therefore it is not surprising that the proof requires a thorough understanding of these subalgebras.

To investigate the subalgebras $\mathcal{B}_{\sigma}$ we define the L-module $\left(\theta_{\sigma}, \mathcal{A}_{\sigma}\right)$, where

$$
\mathcal{A}_{\sigma}=\bigoplus_{(\mu, \lambda) \in \Lambda_{\mathcal{B}}(\sigma)} A^{\lambda / \mu}
$$

The crucial observation is that since we are restricting to a fixed order type there is a natural product on $\mathcal{A}_{\sigma}$. The multiplication

$$
A^{\lambda / \mu} \otimes A^{\lambda^{\prime} / \mu^{\prime}} \rightarrow A^{\lambda+\lambda^{\prime} / \mu+\mu^{\prime}}
$$

is given by Cartan product of irreducible L-modules, which induces an algebra structure on $\mathcal{A}_{\sigma}$ (cf. Lemma 6.1). The algebra $\mathcal{A}_{\sigma}$ is a naturally occurring L-algebra. Indeed, in Lemma 6.2 we show that $\mathcal{A}_{\sigma}$ is isomorphic as a graded L-algebra to the ring of polynomials $\mathcal{O}(\mathrm{V})$ on a certain L-module $\mathrm{V}$.

A priori the algebras $\mathcal{B}_{\sigma}$ and $\mathcal{A}_{\sigma}$ seem to be quite different. Indeed, the product maps $A^{\lambda / \mu} \otimes A^{\lambda^{\prime} / \mu^{\prime}} \rightarrow A^{\lambda+\lambda^{\prime} / \mu+\mu^{\prime}}$ in $\mathcal{A}_{\sigma}$ are surjective, while the Cartan product of multiplicity spaces need not be surjective. For example, consider the case $\lambda=\lambda^{\prime}=(2,1,0), \mu=(2,0)$, and $\mu^{\prime}=(0,0)$. By Corollary 3.3, $\operatorname{dim} W^{\lambda / \mu}=\operatorname{dim} W^{\lambda^{\prime} / \mu^{\prime}}=2$, and $\operatorname{dim} W^{\lambda+\lambda^{\prime} / \mu+\mu^{\prime}}=9$. Therefore the product map cannot be surjective in this case.

Notice that in the above example $(\mu, \lambda)$ and $\left(\mu^{\prime}, \lambda^{\prime}\right)$ do not satisfy a common order type. An important result for us is that if the multiplicity spaces do satisfy a common order type, then their product is surjective. This will be our most crucial application of Theorem 3.1 .

Proposition 3.6. Let $\sigma \in \Sigma$ and let $(\mu, \lambda),\left(\mu^{\prime}, \lambda^{\prime}\right) \in \Lambda_{\mathcal{B}}(\sigma)$. Then the map

$$
W^{\lambda / \mu} \otimes W^{\lambda^{\prime} / \mu^{\prime}} \stackrel{\pi_{\lambda, \lambda^{\prime}}}{\longrightarrow} W^{\lambda+\lambda^{\prime} / \mu+\mu^{\prime}}
$$

is surjective.

By Proposition 3.3, $\mathcal{B}_{\sigma}$ and $\mathcal{A}_{\sigma}$ are isomorphic as $\mathrm{SL}_{2}$ modules. The above proposition shows, moreover, that their products behave similarly. In fact, we have the following theorem:

Proposition 3.7. Let $\sigma \in \Sigma$. Then,

(1) There is an isomorphism of graded $\mathrm{SL}_{2}$-algebras, $\phi_{\sigma}: \mathcal{B}_{\sigma} \rightarrow \mathcal{A}_{\sigma}$, which is unique up to scalars.

(2) By part (1) we can transfer the action of $\mathrm{L}$ on $\mathcal{A}_{\sigma}$ to $\mathcal{B}_{\sigma}$, and the resulting representation, $\left(\Phi_{\sigma}, \mathcal{B}_{\sigma}\right)$, of $\mathrm{L}$ is canonical, i.e. independent of the choice of scalars. 
We now have a family of L-algebras $\left\{\left(\Phi_{\sigma}, \mathcal{B}_{\sigma}\right)\right\}_{\sigma \in \Sigma}$. By showing that the action of $\mathrm{L}$ is well-defined on the intersection of these subalgebras, we obtain the representation $(\Phi, \mathcal{B})$ of $\mathrm{L}$ and prove Theorem 3.5 .

We conclude this section by describing how to use our results to resolve the multiplicities that occur in branching of symplectic groups. Recall that generically the branching of the symplectic groups is not multiplicity free. It's a fundamental problem in classical invariant theory to resolve these multiplicities. Theorem 3.5 provides a solution to this problem that is rooted in classical invariant theoretic techniques. Another solution to this problem using the theory of quantum groups, in particular Yangians, appears in [Mol99].

Now, it is well known that irreducible L-modules have one dimensional weight spaces. Therefore, by Theorem 3.5 we obtain a canonical decomposition of the multiplicity spaces $W^{\lambda / \mu}$ into one-dimensional spaces. A priori, it seems that this decomposition depends on a choice of torus of $\mathrm{L}$. We will show that in fact this choice is induced by the torus of $\mathrm{Sp}_{2 \mathrm{n}}$ that is fixed throughout. More precisely, we have:

Corollary 3.8. Let $(\mu, \lambda) \in \Lambda$ There is a canonical decomposition of $W^{\lambda / \mu}$ into one dimensional spaces

$$
W^{\lambda / \mu}=\bigoplus_{\substack{\gamma \in \Lambda_{n} \\ \mu<\gamma<\lambda^{+}}} W^{\lambda / \gamma / \mu} .
$$

In particular, $\mathcal{B}$ has a basis which is unique up to scalar.

Properties of the basis of $\mathcal{B}$ appearing in Corollary 3.8 are studied in [KY]; in particular we show that it is a standard monomial basis, i.e. it satisfies a straightening law. We then use that to describe an explicit toric deformation of $\operatorname{Spec}(\mathcal{B})$.

\section{Proof of TheOREM 3.1}

4.1. Some results of Zhelobenko. Let $G$ be a connected classical group. We use freely the notation from Section 2.1. Let $\lambda \in \Lambda_{\mathrm{G}}$. Then $\mathrm{F}_{\mathrm{G}}^{\lambda} \subset \mathcal{R}_{\mathrm{G}}$ embeds linearly in $\mathcal{O}\left(\mathrm{U}_{\mathrm{G}}\right)$ via res : $\left.\mathrm{f} \mapsto f\right|_{\mathrm{U}_{\mathrm{G}}}$. Set $\mathrm{Z}_{\lambda}\left(\mathrm{U}_{\mathrm{G}}\right)=\operatorname{res}_{\mathrm{U}_{\mathrm{G}}}\left(\mathrm{F}_{\mathrm{G}}^{\lambda}\right)$. If there is no cause for confusion, we write simply $Z_{\lambda}=Z_{\lambda}\left(U_{G}\right)$.

We define a representation of $G$ on $Z_{\lambda}$ as follows. Let $e^{\lambda}: T_{G} \rightarrow \mathbb{C}$ be the character of $T_{G}$ given by $t \mapsto t^{\lambda}$. We extend this character to $\bar{U}_{G} T_{G} U_{G}$ by defining $e^{\lambda}(\bar{u} t u)=t^{\lambda}$. Then by continuity $e^{\lambda}$ is defined on all of $G$. Now let $u \in U_{G}, g \in u^{-1} \bar{U}_{G} T_{G} U_{G}$, and $\mathrm{f} \in \mathrm{Z}_{\lambda}$. Write $\mathrm{ug}=\overline{\mathrm{u}}_{1} \mathrm{t}_{1} \mathrm{u}_{1} \in \overline{\mathrm{U}}_{\mathrm{G}} \mathrm{T}_{\mathrm{G}} \mathrm{U}_{\mathrm{G}}$. Then define

$$
g . f(u)=e^{\lambda}\left(t_{1}\right) f\left(u_{1}\right) \text {. }
$$

Since $\mathrm{u}^{-1} \overline{\mathrm{U}}_{\mathrm{G}} \mathrm{T}_{\mathrm{G}} \mathrm{U}_{\mathrm{G}}$ is dense, we extend this action to all of $\mathrm{G}$. Note that the constant function $z_{\lambda}: u \mapsto 1$ is a canonical highest weight vector in $Z_{\lambda}$ of weight $\lambda$, and res $: F_{G}^{\lambda} \rightarrow$ $Z_{\lambda}$ is an isomorphism of G-modules.

Let $\left\{\alpha_{1}, \ldots, \alpha_{n}\right\}$ be a set of simple roots relative to the positive roots $\Phi^{+}$. For each $\alpha_{i}$ choose a nonzero root vector $X_{i} \in \mathfrak{g}_{\alpha_{i}}$. Let $\mathrm{D}_{i}$ be the differential operator on $\mathcal{O}\left(\mathrm{U}_{\mathrm{G}}\right)$ corresponding to the infinitesimal action of $X_{i}$ acting on $\mathcal{O}\left(\mathrm{U}_{\mathrm{G}}\right)$ by left translation. 
Finally, let $\left\{\varpi_{1}, \ldots, \varpi_{n}\right\}$ be the fundamental weights and suppose $\lambda=m_{1} \varpi_{1}+\cdots+$ $m_{n} \varpi_{n} \in \Lambda_{G}$.

Proposition 4.1 (Theorem 1, §65, Chapter $\mathrm{X}$, [Zh73]). The space $\mathrm{Z}_{\lambda} \subset \mathcal{O}\left(\mathrm{U}_{\mathrm{G}}\right)$ is the solutions to the system of differential equations $\left\{D_{i}^{m_{i}+1}=0: i=1, \ldots, n\right\}$. In other words,

$$
Z_{\lambda}=\left\{f \in \mathcal{O}\left(U_{G}\right): D_{i}^{m_{i}+1} f=0 \text { for } i=1, \ldots, n\right\} .
$$

In [Zh73] the system of differential equations $\left\{D_{i}^{m_{i}+1}=0: i=1, \ldots, n\right\}$ is termed the "indicator system". Notice that by the Leibniz rule $Z_{\lambda} Z_{\lambda^{\prime}}=Z_{\lambda+\lambda^{\prime}}$.

Consider the ring $\mathcal{O}\left(\mathrm{U}_{\mathrm{G}} \times \mathbb{C}^{n}\right)$. Let $t_{1}, \ldots, t_{\mathrm{n}}$ be the standard coordinates on $\mathbb{C}^{\mathrm{n}}$. Then $\mathcal{O}\left(\mathrm{U}_{\mathrm{G}} \times \mathbb{C}^{\mathrm{n}}\right)=\bigoplus_{\overline{\mathrm{m}} \in \mathbb{N}^{n}} \mathcal{O}\left(\mathrm{U}_{\mathrm{G}}\right) \otimes \mathrm{t}_{1}^{\mathrm{m}_{1}} \ldots \mathrm{t}_{\mathrm{n}}^{\mathrm{m}_{\mathrm{n}}}$. Set $\bar{m}_{\lambda}=\left(\mathrm{m}_{1}, \ldots, \mathrm{m}_{\mathrm{n}}\right)$ where $\lambda=$ $m_{1} \varpi_{1}+\cdots+m_{n} \varpi_{n}$. We form the subring

$$
\mathcal{Z}_{\mathrm{G}}=\bigoplus_{\lambda \in \Lambda_{\mathrm{G}}} \mathrm{Z}_{\lambda} \otimes \mathrm{t}^{\overline{\mathrm{m}}_{\lambda}}
$$

of $\mathcal{O}\left(\mathrm{U}_{\mathrm{G}} \times \mathbb{C}^{\mathfrak{n}}\right)$. This is a G-ring, with $\mathrm{G}$ acting on the left factor. Define a map

$$
\text { res }: \mathcal{R}_{\mathrm{G}} \rightarrow \mathcal{Z}_{\mathrm{G}}
$$

by

$$
\left.f \in F_{G}^{\lambda} \mapsto f\right|_{U_{G}} \otimes t^{\bar{m}_{\lambda}} \in Z_{\lambda} \otimes t^{\bar{m}_{\lambda}} .
$$

It's easy to see that:

Proposition 4.2. The map res : $\mathcal{R}_{\mathrm{G}} \rightarrow \mathcal{Z}_{\mathrm{G}}$ is an isomorphism of $\mathrm{G}$-rings.

4.2. Preparatory lemmas. We now specialize the results of the previous section to our setting.

For $\lambda \in \Lambda_{n}$ we consider Zhelobenko's realization of $V^{\lambda^{+}}$, which is denoted $Z_{\lambda^{+}}\left(U_{n+1}\right)$. So $Z_{\lambda^{+}}\left(U_{n+1}\right)$ is an irreducible $G_{n+1}$-module. Let $x_{i j}$ be the standard coordinates on $\mathrm{U}_{\mathrm{n}+1}$.

Zhelobenko's realization of the irreducible $S p_{2 n}$-module of highest weight $\lambda$ is denoted $Z_{\lambda}\left(U_{C_{n}}\right)$. For the affine space $U_{C_{n}}$, the following can be taken as coordinates:

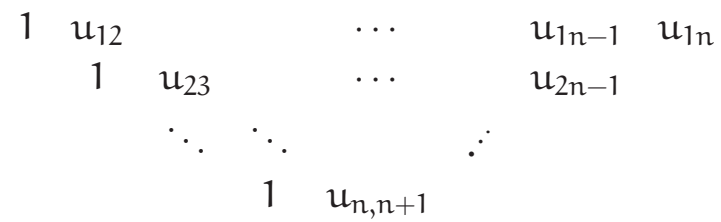

(The one's are retained here in order to preserve the symmetry of the entries.) The other entries of $U_{C_{n}}$ are polynomials in these coordinates.

The group $\mathrm{U}_{n-1}$ acts on $\mathcal{O}\left(\mathrm{U}_{n+1}\right)$ by right translation, and a straight-forward calculation shows that $f \in \mathcal{O}\left(\mathrm{U}_{n+1}\right)^{\mathrm{u}_{n-1}}$ if, and only if, it's a polynomial in the variables

$$
\left\{x_{i, j}: i=1, \ldots, n j=n, n+1 \text { and } i<j\right\} \text {. }
$$

Similarly, $\mathcal{O}\left(\mathrm{U}_{\mathrm{C}_{n}}\right)^{\mathrm{U}_{\mathrm{C}_{n-1}}}$ is the polynomial ring in the variables

$$
\left\{u_{i, j}: i=1, \ldots, n j=n, n+1 \text { and } i<j\right\} .
$$


Hence both $\mathcal{O}\left(\mathrm{U}_{n+1}\right)^{\mathrm{u}_{n-1}}$ and $\mathcal{O}\left(\mathrm{U}_{\mathrm{C}_{n}}\right)^{\mathrm{u}_{\mathrm{C}_{n-1}}}$ are polynomial rings in $2 \mathrm{n}-1$ variables.

Let $\psi_{a, b}: M_{m, n} \rightarrow M_{a, b}$ be the map assigning a matrix its principal $a \times b$ submatrix, and set $\psi_{a}=\psi_{a, a}$. Let $\psi_{z}=\left.\psi_{n+1}\right|_{u_{n}}: U_{C_{n}} \rightarrow U_{n+1}$. The induced map on functions $\psi_{Z}^{*}: \mathcal{O}\left(u_{n+1}\right) \rightarrow \mathcal{O}\left(u_{C_{n}}\right)$ satisfies $\psi_{Z}^{*}\left(x_{i j}\right)=u_{i j}$. By our descriptions of the rings $\mathcal{O}\left(\mathrm{U}_{\mathrm{C}_{n}}\right)^{\mathrm{u}_{\mathrm{C}_{n-1}}}$ and $\mathcal{O}\left(\mathrm{U}_{n+1}\right)^{\mathrm{u}_{n-1}}, \psi_{Z}^{*}: \mathcal{O}\left(\mathrm{U}_{n+1}\right)^{\mathrm{u}_{n-1}} \rightarrow \mathcal{O}\left(\mathrm{U}_{\mathrm{C}_{n}}\right)^{\mathrm{u}_{\mathrm{C}_{n-1}}}$ is a ring isomorphism.

Lemma 4.3. Let $\lambda \in \Lambda_{n}$. The map $\psi_{Z}^{*}$ restricts to a linear isomorphism:

$$
\psi_{Z}^{*}: Z_{\lambda^{+}}\left(\mathrm{U}_{n+1}\right)^{\mathrm{U}_{n-1}} \stackrel{\simeq}{\rightarrow} \mathrm{Z}_{\lambda}\left(\mathrm{U}_{\mathrm{C}_{n}}\right)^{\mathrm{U}_{\mathrm{C}_{n-1}}}
$$

Proof. Let $\bar{m}_{\lambda}=\left(m_{1}, \ldots, m_{n}\right)$. In the proof of Theorem 4, $\S 114$ in [Zh73], Zhelobenko shows that $\mathrm{Z}_{\lambda}\left(\mathrm{U}_{\mathrm{C}_{n}}\right)^{\mathrm{U}_{\mathrm{C}_{n-1}}}$ equals

$$
\left\{f \in \mathcal{O}\left(u_{C_{n}}\right)^{u_{c_{n-1}}}:\left(u_{i+1, n} \frac{\partial}{\partial u_{i, n}}+u_{i+1, n+1} \frac{\partial}{\partial u_{i, n+1}}\right)^{m_{i}+1}(f)=0 \text { for } i=1, \ldots, n\right\}
$$

while $Z_{\lambda^{+}}\left(U_{n+1}\right)^{u_{n-1}}$ equals

$$
\left\{f \in \mathcal{O}\left(u_{n+1}\right)^{u_{n-1}}:\left(x_{i+1, n} \frac{\partial}{\partial x_{i, n}}+x_{i+1, n+1} \frac{\partial}{\partial x_{i, n+1}}\right)^{m_{i}+1}(f)=0 \text { for } i=1, \ldots, n\right\} .
$$

With these descriptions in hand, and the fact that $\psi_{Z}^{*}\left(x_{i j}\right)=u_{i j}$, it follows that

$$
\psi_{Z}^{*}\left(Z_{\lambda^{+}}\left(U_{n+1}\right)^{u_{n-1}}\right)=Z_{\lambda}\left(U_{C_{n}}\right)^{u_{C_{n-1}}} .
$$

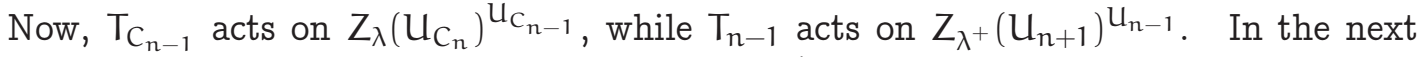
lemma, these tori are both identified with $\left(\mathbb{C}^{\times}\right)^{n-1}$.

Lemma 4.4. Let $\lambda \in \Lambda_{n}$. Then $\psi_{Z}^{*}: Z_{\lambda^{+}}\left(U_{n+1}\right)^{\mathrm{U}_{n-1}} \rightarrow \mathrm{Z}_{\lambda}\left(\mathrm{U}_{\mathrm{C}_{n}}\right)^{\mathrm{U}_{\mathrm{C}_{n-1}}}$ is a $\left(\mathbb{C}^{\times}\right)^{\mathrm{n}-1}$ isomorphism.

Proof. By Lemma 4.3, it remains to show only that $\psi_{Z}^{*}$ intertwines the $\left(\mathbb{C}^{\times}\right)^{n-1}$-action.

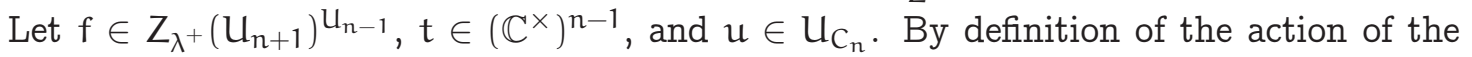
tori (cf. (99)),

$$
\psi_{Z}^{*}(t . f)(u)=e^{\lambda}(t) f\left(t^{-1} \psi_{Z}(u) t\right)
$$

while

$$
\text { t. } \psi_{Z}^{*}(f)(u)=e^{\lambda}(t) f\left(\psi_{Z}\left(t^{-1} u t\right)\right) .
$$

It's easy to check now that $\psi_{Z}^{*}(t . f)(u)=t . \psi_{Z}^{*}(f)(u)$.

For $\mu \in \Lambda_{n-1}$ let $Z_{\lambda / \mu}\left(U_{C_{n}}\right)$ (resp. $Z_{\lambda^{+} / \mu}\left(U_{n+1}\right)$ ) denote the $\mu$ weight spaces of $Z_{\lambda}\left(U_{C_{n}}\right)^{U_{C_{n-1}}}\left(\right.$ resp. $\left.Z_{\lambda^{+}}\left(U_{n+1}\right)^{U_{n-1}}\right)$. Lemma 4.4 implies that

$$
\psi_{\mathrm{Z}}^{*}: \mathrm{Z}_{\lambda^{+} / \mu}\left(\mathrm{U}_{\mathrm{n}+1}\right) \stackrel{\simeq}{\rightrightarrows} \mathrm{Z}_{\lambda / \mu}\left(\mathrm{U}_{\mathrm{C}_{\mathrm{n}}}\right)
$$

is a linear isomorphism. These spaces are isomorphic to the multiplicity spaces $\mathrm{V}^{\lambda^{+} / \mu}$ and $W^{\lambda / \mu}$. Recall that $S L_{2}$ acts on these multiplicity spaces. 
Lemma 4.5. Let $\lambda \in \Lambda_{n}$ and $\mu \in \Lambda_{n-1}$. Then

$$
\psi_{Z}^{*}: Z_{\lambda^{+} / \mu}\left(\mathrm{U}_{n+1}\right) \rightarrow \mathrm{Z}_{\lambda / \mu}\left(\mathrm{U}_{\mathrm{C}_{\mathrm{n}}}\right)
$$

is an $\mathrm{SL}_{2}$-isomorphism.

Proof. As mentioned above, the map $\psi_{Z}^{*}: Z_{\lambda^{+} / \mu}\left(U_{n+1}\right) \rightarrow Z_{\lambda / \mu}\left(U_{C_{n}}\right)$ is a linear isomorphism, so we just need to show it intertwines the $\mathrm{SL}_{2}$ action. Now, the action of of $\mathrm{SL}_{2}$ on the multiplicity spaces is defined via its embeddings in $G_{n+1}$ and $S p_{2 n}$ as explained in Section 2.2. For the sake of clarity, let us denote these embeddings by $\alpha: \mathrm{SL}_{2} \hookrightarrow \mathrm{GL}_{n+1}$ and $\beta: S L_{2} \hookrightarrow S p_{2 n}$. Note that for $x \in S L_{2}$, we have $\psi_{n+1}(\beta(x))=\alpha(x)$.

Let $f \in Z_{\lambda^{+}}\left(U_{n+1}\right)^{u_{n-1}}, x \in S L_{2}$, and $u \in U_{C_{n}}$. We want to show that

$$
\psi_{n+1}^{*}(\alpha(x) . f)(u)=\left(\beta(x) \cdot\left(\psi_{n+1}^{*}(f)\right)\right)(u) .
$$

By definition of the action of $S p_{2 n}$ on $Z_{\lambda}\left(U_{C_{n}}\right)$ (see (9)), we have

$$
\left(\beta(x) \cdot\left(\psi_{n+1}^{*}(f)\right)\right)(u)=e^{\lambda}\left(t_{1}\right) f\left(\psi_{n+1}\left(u_{1}\right)\right),
$$

where

$$
u \beta(x)=\bar{u}_{1} t_{1} u_{1} \in \bar{u}_{C_{n}} T_{C_{n}} u_{C_{n}} .
$$

To describe the left hand side of (13), we need to first decompose $\psi_{n+1}(u) \alpha(x)$ into a product compatible with $\overline{\mathrm{U}}_{n+1} \mathrm{~T}_{n+1} \mathrm{U}_{n+1}$. To wit,

$$
\begin{aligned}
\psi_{n+1}(u) \alpha(x) & =\psi_{n+1}(u) \psi_{n+1}(\beta(x)) \\
& =\psi_{n+1}(u \beta(x)) \\
& =\psi_{n+1}\left(\bar{u}_{1} t_{1} u_{1}\right) \\
& =\psi_{n+1}\left(\bar{u}_{1}\right) \psi_{n+1}\left(t_{1}\right) \psi_{n+1}\left(u_{1}\right) .
\end{aligned}
$$

Therefore

$$
\psi_{n+1}^{*}(\alpha(x) \cdot f)(u)=e^{\lambda^{+}}\left(\psi_{n+1}\left(t_{1}\right)\right) f\left(\psi_{n+1}\left(u_{1}\right)\right) .
$$

Since clearly, $e^{\lambda}\left(t_{1}\right)=e^{\lambda^{+}}\left(\psi_{n+1}\left(t_{1}\right)\right)$, (13) holds.

4.3. Proof of Theorem 3.1. Recall the homomorphism $\psi^{*}: \mathcal{O}\left(M_{n, n+1}\right) \rightarrow \mathcal{O}\left(S p_{2 n}\right)$ (see (7)), which is induced from the map $\psi$ taking an element of $S p_{2 n}$ to its principal $n \times(n+1)$ submatrix.

Lemma 4.6. We have $\psi^{*}\left(\mathcal{B}^{\prime}\right) \subset \mathcal{B}$. Moreover, $\psi^{*}: \mathcal{B}^{\prime} \rightarrow \mathcal{B}$ is an $\Lambda_{n-1, n \text {-graded map }}$ of $\mathrm{SL}_{2}$ algebras.

Proof. Let $\mathrm{f} \in \mathcal{B}^{\prime}, \overline{\mathrm{u}} \in \overline{\mathrm{U}}_{\mathrm{C}_{n}}, \mathrm{~g} \in \mathrm{Sp}_{2 \mathrm{n}}$, and $\mathrm{u} \in \mathrm{U}_{\mathrm{C}_{n-1}}$. We must show that $\mathrm{f}(\psi(\overline{\mathrm{u}} g u))=$ $f(\psi(g))$. Indeed, a straight-forward computation using block matrices shows that

$$
\psi(\bar{u} g u)=\psi_{n}(\bar{u}) \psi(g) \psi_{n+1}(u) .
$$

Since clearly $\psi_{n}(\bar{u}) \in \overline{\mathrm{u}}_{\mathrm{n}}$ and $\psi_{n+1}(\mathrm{u}) \in \mathrm{U}_{n-1}$, the first statement follows. 
Now suppose $f \in V^{\lambda^{+} / \mu}$. Let $t \in T_{C_{n}}, s \in T_{C_{n-1}}$, and $g \in S p_{2 n}$. Then

$$
\begin{aligned}
(t, s) \cdot \psi^{*}(f)(g) & =\psi^{*}(f)\left(t^{-1} g s\right) \\
& =f\left(\psi\left(t^{-1} g s\right)\right) \\
& =f\left(\psi_{n}\left(t^{-1}\right) \psi(g) \psi_{n-1}(s)\right) \\
& =\psi_{n}(t)^{-\lambda} \psi_{n-1}(s)^{\mu} f(\psi(g)) \\
& =t^{-\lambda} s^{\mu} f(\psi(g)) \\
& =t^{-\lambda} s^{\mu} \cdot \psi^{*}(f)(g) .
\end{aligned}
$$

Therefore $\psi^{*}(f) \in W^{\lambda / \mu}$, and hence $\psi^{*}$ is graded.

Finally, we must show $\psi^{*}$ intertwines the $S L_{2}$-action. Indeed, let $f \in \mathcal{B}^{\prime}, x \in S L_{2}$, and $g \in S p_{2 n}$. Then another computation with block matrices shows that $f(\psi(g) x)=$ $f(\psi(g x))$. (Here one has to be careful to use the correct embeddings of $S L_{2}$ in $G L_{n+1}$ and $S p_{2 n}$ that define the corresponding actions.) Therefore, $x \cdot \psi^{*}(f)=\psi^{*}(x . f)$.

By the above lemma, $\psi^{*}: \mathcal{B}^{\prime} \rightarrow \mathcal{B}$ is a morphism of $\Lambda_{n-1, n}$-graded algebras. To complete the proof of Theoreme 3.1 we must it is an isomorphism.

Let $U_{n, n+1}=\psi_{n, n+1}\left(u_{n+1}\right)$. We identify the affine spaces $U_{n+1}$ and $u_{n, n+1}$ in the obvious way. For $\lambda \in \Lambda_{n}$, consider the embedding $Z_{\lambda^{+}}\left(u_{n+1}\right) \hookrightarrow \mathcal{O}\left(u_{n, n+1}\right)$. Denote the image of this embedding by $Z_{\lambda^{+}}\left(U_{n, n+1}\right)$. (Similarly, denote by $Z_{\lambda^{+} / \mu}\left(U_{n, n+1}\right)$ the image of $Z_{\lambda^{+} / \mu}\left(U_{n+1}\right)$ under the embedding.) By transfer of structure, $Z_{\lambda^{+}}\left(U_{n, n+1}\right)$ is an irreducible $\mathrm{GL}_{\mathfrak{n}+1}$-module of highest weight $\lambda^{+}$, i.e. we decree that

$$
\psi_{n, n+1}^{*}: Z_{\lambda^{+}}\left(U_{n, n+1}\right) \rightarrow Z_{\lambda^{+}}\left(U_{n+1}\right)
$$

is an isomorphism of $\mathrm{GL}_{n+1}$-modules. Of course, the inverse of this isomorphism is the map induced by the embedding $\phi: \mathrm{U}_{n, n+1} \rightarrow \mathrm{U}_{n+1}$. Combining this isomorphism with Lemma 4.5 we obtain that

$$
\psi_{n, n+1}^{*}: Z_{\lambda^{+} / \mu}\left(U_{n, n+1}\right) \rightarrow Z_{\lambda / \mu}\left(U_{C_{n}}\right)
$$

is an isomorphism of $\mathrm{SL}_{2}$-modules.

Consider now

$$
\mathcal{B}_{Z}^{\prime}=\bigoplus_{(\mu, \lambda) \in \Lambda_{n-1, n}} Z_{\lambda^{+} / \mu}\left(U_{n, n+1}\right) \otimes t^{\bar{m}_{\lambda^{+}}}
$$

and

$$
\mathcal{B}_{Z}=\bigoplus_{(\mu, \lambda) \in \Lambda_{n-1, n}} Z_{\lambda / \mu}\left(U_{C_{n}}\right) \otimes t^{\bar{m}_{\lambda}}
$$

These are subalgebras of $\mathcal{Z}_{\mathrm{GL}_{n+1}}$ and $\mathcal{Z}_{\mathrm{Sp}_{2 n}}$, respectively (see (10)).

We define a map $\mathcal{B}_{Z}^{\prime} \rightarrow \mathcal{B}_{Z}$ which on graded components is simply given by

$$
\psi_{n, n+1}^{*} \otimes 1: Z_{\lambda^{+} / \mu}\left(U_{n, n+1}\right) \otimes t^{\bar{m}_{\lambda^{+}}} \rightarrow Z_{\lambda / \mu}\left(U_{C_{n}}\right) \otimes t^{\bar{m}_{\lambda}} .
$$

Let us denote the total map by $\psi_{n, n+1}^{*} \otimes 1: \mathcal{B}_{Z}^{\prime} \rightarrow \mathcal{B}_{Z}$ also.

By (14) $\psi_{n, n+1}^{*} \otimes 1: \mathcal{B}_{Z}^{\prime} \rightarrow \mathcal{B}_{Z}$ is a isomorphism of $\mathrm{SL}_{2}$-modules. But clearly the map is a morphism of graded algebras, so in fact it is an isomorphism of $\Lambda_{n-1, n}$-graded $\mathrm{SL}_{2}$-algebras. Now, by Proposition 4.2 , the restriction of res : $\mathcal{R}_{\mathrm{GL}_{n+1}} \rightarrow \mathcal{Z}_{\mathrm{GL}_{n+1}}$ to $\mathcal{B}^{\prime}$ 
gives an isomorphism res : $\mathcal{B}^{\prime} \rightarrow \mathcal{B}_{Z}^{\prime}$ of $\Lambda_{n-1, n}$-graded $\mathrm{SL}_{2}$-algebras. Similarly, we have the isomorphism res $: \mathcal{B} \rightarrow \mathcal{B}_{Z}$ of $\Lambda_{n-1, n}$-graded $\mathrm{SL}_{2}$-algebras.

We now have a diagram:

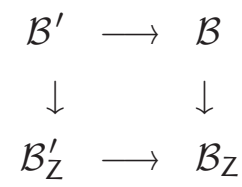

where the vertical arrows are given by res, the bottom arrow is $\psi_{n, n+1}^{*} \otimes 1$, and the top arrow is $\psi^{*}$. Clearly, this diagram commutes. Indeed, this follows from the simple fact that for $f \in \mathcal{O}\left(M_{n, n+1}\right)$,

$$
\left.(f \circ \psi)\right|_{\mathrm{c}_{\mathrm{n}}}=\left.f\right|_{\mathrm{u}_{n, n+1}} \circ \psi_{\mathrm{n}, \mathrm{n}+1}
$$

as elements of $\mathcal{O}\left(\mathrm{U}_{\mathrm{C}_{\mathrm{n}}}\right)$. Moreover, by the previous paragraph the bottom three maps are isomorphisms of $\Lambda_{n-1, n}$-graded $\mathrm{SL}_{2}$-algebras by. We conclude that $\psi^{*}: \mathcal{B}^{\prime} \rightarrow \mathcal{B}$ is an isomorphism of $\Lambda_{n-1, n}$-graded $S L_{2}$-algebras, thus completing the proof of Theorem 3.1 .

\section{Proof of Proposition 3.6}

In this section we prove various structural results about the multiplicity spaces $V_{\lambda / \mu}$ where $(\mu, \lambda) \in \Lambda_{n-1, n+1}$. Notice that these are multiplicity spaces that occur in branching from $G L_{n+1}$ to $G L_{n-1}$. By virtue of Theorem 3.1, these results have analogous in the setting of branching of symplectic groups, and it is for this reason that these results are important for us.

We will work in slightly greater generality than strictly necessary, and consider the semigroup

$$
\begin{aligned}
\Omega & =\left\{(\mu, \lambda) \in \Lambda_{n-1, n+1}: V_{\lambda / \mu} \neq\{0\}\right\} \\
& =\left\{(\mu, \lambda) \in \Lambda_{n-1, n+1}: \mu \ll \lambda\right\} .
\end{aligned}
$$

The second equality follows from (3).

5.1. The rearrangement function. We begin by introducing the rearrangement function on $\Omega$. Define $f: \Omega \rightarrow \Lambda_{2 n}$ by:

$$
(\mu, \lambda) \stackrel{f}{\mapsto}\left(x_{1}, y_{1}, \ldots, x_{n}, y_{n}\right)
$$

where $\left\{x_{1} \geq y_{1} \geq \cdots \geq x_{n} \geq y_{n}\right\}$ is the non-increasing rearrangement of $\left(\mu_{1}, \ldots, \mu_{n-1}, \lambda_{1}, \ldots, \lambda_{n+1}\right)$. Notice that $f(\mu, \lambda)$ equals

$$
\left(\lambda_{1} \geq \max \left(\mu_{1}, \lambda_{2}\right) \geq \min \left(\mu_{1}, \lambda_{2}\right) \geq \cdots \geq \max \left(\mu_{n-1}, \lambda_{n}\right) \geq \min \left(\mu_{n-1}, \lambda_{n}\right) \geq \lambda_{n+1}\right) .
$$

This easily implies:

Lemma 5.1. Let $(\mu, \lambda) \in \Omega$. Suppose $f(\mu, \lambda)=\left(x_{1}, y_{1}, \ldots, x_{n}, y_{n}\right)$ and $\gamma \in \Lambda_{n}$. Then $\mu<\gamma<\lambda$ if, and only if, $y_{i} \leq \gamma_{i} \leq x_{i}$ for $i=1, \ldots, n$, where $\gamma=\left(\gamma_{1}, \ldots, \gamma_{n}\right)$.

For $\sigma \in \Sigma$ let $\Omega(\sigma)$ be the sub-semigroup of $\Omega$ consisting of the pairs of order type $\sigma$. Let $f_{\sigma}$ denote the restriction of $f$ to $\Omega(\sigma)$.

Lemma 5.2. Let $\sigma \in \Sigma$. Then $\mathrm{f}_{\sigma}: \Omega(\sigma) \rightarrow \Lambda_{2 n}$ is a semigroup isomorphism. 
Proof. For $(\mu, \lambda) \in \Omega(\sigma)$ let $f_{\sigma}(\mu, \lambda)=\left(f_{\sigma}(\mu, \lambda)_{1}, \ldots, f_{\sigma}(\mu, \lambda)_{2 n}\right)$. Define functions

$$
\begin{aligned}
& a:\{1, \ldots, n-1\} \rightarrow\{1, \ldots, 2 n\} \\
& b:\{1, \ldots, n+1\} \rightarrow\{1, \ldots, 2 n\}
\end{aligned}
$$

by $b(1)=1$ and $b(n+1)=2 n$, and for $i=1, \ldots, n-1$

$$
\begin{aligned}
& \left(\sigma_{i} \text { is } \geq\right) \Longrightarrow a(i)=2 i \text { and } b(i+1)=2 i+1 \\
& \left(\sigma_{i} \text { is } \leq\right) \Longrightarrow a(i)=2 i+1 \text { and } b(i+1)=2 i
\end{aligned}
$$

Then for all $(\mu, \lambda) \in \Omega(\sigma)$

$$
\begin{aligned}
& \mu_{i}=f_{\sigma}(\mu, \lambda)_{a(i)} \text { for } i=1, \ldots, n-1 \\
& \lambda_{j}=f_{\sigma}(\mu, \lambda)_{b(j)} \text { for } j=1, \ldots, n+1 .
\end{aligned}
$$

This implies that $f_{\sigma}$ is an injective semigroup homomorphism.

Now suppose $\left(z_{1}, \ldots, z_{2 n}\right) \in \Lambda_{2 n}$ is given. Define $\mu$ and $\lambda$ by the formulas

$$
\begin{aligned}
& \mu_{i}=z_{a(i)} \text { for } i=1, \ldots, n-1 \\
& \lambda_{j}=z_{b(j)} \text { for } j=1, \ldots, n+1 .
\end{aligned}
$$

Since $a(1)<a(2)<\cdots<a(n-1)$, it follows that $\mu \in \Lambda_{n-1}$. Similarly, $\lambda \in \Lambda_{n+1}^{+}$. Since $b(\mathfrak{i})<a(i)<b(i+2)$, we get that $\mu \ll \lambda$. Finally, suppose $\sigma_{i}$ is $\geq$. Then $a(i)<b(i+1)$, and so $\mu_{i} \geq \lambda_{i+1}$. Similarly, if $\sigma_{i}$ is $\leq$ then $\mu_{i} \leq \lambda_{i+1}$. Therefore $(\mu, \lambda) \in \Omega(\sigma)$. Since $f_{\sigma}(\mu, \lambda)=\left(z_{1}, \ldots, z_{2 n}\right)$ we conclude that $f_{\sigma}$ is surjective.

Lemma 5.3. Let $\sigma \in \Sigma$ and let $(\mu, \lambda),\left(\mu^{\prime}, \lambda^{\prime}\right) \in \Omega(\sigma)$. Suppose that $\gamma \in \Lambda_{n}$ satisfies

$$
\mu+\mu^{\prime}<\gamma<\lambda+\lambda^{\prime}
$$

Then there exist $\nu, v^{\prime} \in \Lambda_{n}$ such that $\gamma=v+v^{\prime}, \mu<v<\lambda$, and $\mu^{\prime}<v^{\prime}<\lambda^{\prime}$.

Proof. Set $f_{\sigma}(\mu, \lambda)=\left(x_{1}, y_{1}, \ldots, x_{n}, y_{n}\right)$ and $f_{\sigma}\left(\mu^{\prime}, \lambda^{\prime}\right)=\left(x_{1}^{\prime}, y_{1}^{\prime}, \ldots, x_{n}^{\prime}, y_{n}^{\prime}\right)$. By Lemma 5.2 ,

$$
f_{\sigma}\left(\mu+\mu^{\prime}, \lambda+\lambda^{\prime}\right)=\left(x_{1}+x_{1}^{\prime}, y_{1}+y_{1}^{\prime}, \ldots, x_{n}+x_{n}^{\prime}, y_{n}+y_{n}^{\prime}\right) .
$$

Therefore by Lemma 5.1, $y_{i}+y_{i}^{\prime} \leq \gamma_{i} \leq x_{i}+x_{i}^{\prime}$. Now choose $v_{i}, v_{i}^{\prime}$ such that $\gamma_{i}=v_{i}+v_{i}^{\prime}$, $y_{i} \leq v_{i} \leq x_{i}$, and $y_{i}^{\prime} \leq v_{i}^{\prime} \leq x_{i}^{\prime}$. Set $v=\left(v_{1}, \ldots, v_{n}\right)$ and $v^{\prime}=\left(v_{1}^{\prime}, \ldots, v_{n}^{\prime}\right)$. Clearly $v, v^{\prime} \in \Lambda_{n}$ and $\gamma=v+v^{\prime}$. Moreover, by Lemma 5.1, $\mu<v<\lambda$, and $\mu^{\prime}<v^{\prime}<\lambda^{\prime}$.

5.2. Proof of Proposition 3.2. For $(\mu, \lambda) \in \Omega$ recall that $r_{i}(\mu, \lambda)=x_{i}-y_{i}$, where $f(\mu, \lambda)=\left(x_{1}, y_{1}, \ldots, x_{n}, y_{n}\right)$. Let $(\mu, \lambda) \in \Lambda_{n-1, n+1}$. Then as a $G_{1} \times G_{1}$-module,

$$
V^{\lambda / \mu} \cong \bigoplus_{\substack{\gamma \in \Lambda_{n} \\ \mu<\gamma<\lambda}} V^{\gamma / \mu} \otimes V^{\lambda / \gamma}
$$

Now, $d(q)=\left(\begin{array}{cc}q & 0 \\ 0 & q^{-1}\end{array}\right)$ acts on $V^{\gamma / \mu} \otimes V^{\lambda / \gamma}$ by the scalar $q^{2|\gamma|-|\lambda|-|\mu|}$. Moreover, by (2) and (3), $\operatorname{dim} V^{\gamma / \mu} \otimes V^{\lambda / \gamma}=1$ for $\gamma$ such that $\mu<\gamma<\lambda$. Therefore the character 
equals

$$
\operatorname{ch}\left(V^{\lambda / \mu}\right)=\sum_{\substack{\gamma \in \Lambda_{n} \\ \mu<\gamma<\lambda}} q^{2|\gamma|-|\lambda|-|\mu|} .
$$

Set $f(\mu, \lambda)=\left(x_{1}, y_{1}, \ldots, x_{n}, y_{n}\right)$ and $r_{i}=r_{i}(\mu, \lambda)$. By Lemma 5.1,

$$
\begin{aligned}
\sum_{\substack{\gamma \in \Lambda_{n} \\
\mu<\gamma<\lambda}} q^{2|\gamma|-|\lambda|-|\mu|} & =\sum_{0 \leq j_{i} \leq r_{i}} q^{2\left(y_{1}+\cdots+y_{n}+j_{1}+\cdots+j_{n}\right)-\left(x_{1}+\cdots+x_{n}+y_{1}+\cdots+y_{n}\right)} \\
& =\sum_{0 \leq j_{i} \leq r_{i}} q^{\left(-r_{1}+2 j_{1}\right)+\cdots+\left(-r_{n}+2 j_{n}\right)} \\
& =\prod_{i=1}^{n} \sum_{j=0}^{r_{i}} q^{-r_{i}+2 j} \\
& =\prod_{i=1}^{n} \operatorname{ch}\left(F^{r_{i}}\right)
\end{aligned}
$$

Therefore $\operatorname{ch}\left(\mathrm{V}^{\lambda / \mu}\right)=\operatorname{ch}\left(\bigotimes_{i=1}^{n} \mathrm{~F}^{r_{i}(\mu, \lambda)}\right)$. This proves Proposition 3.2 .

5.3. A technical lemma. Suppose $(\gamma, \lambda) \in \Lambda_{n, n+1}$. We may view $V^{\lambda}$ as a $G L_{n}$-module by restriction, and, as such, define $\mathrm{V}^{\lambda}[\gamma]$ to be the $\gamma$-isotypic component of $\mathrm{V}^{\lambda}$. Let $\mathrm{p}_{\gamma}^{\lambda}: \mathrm{V}^{\lambda} \rightarrow \mathrm{V}^{\lambda}[\gamma]$ be the corresponding projection.

Before stating and proving the lemma we first make a simple observation. Suppose $\Theta$ is a semigroup and $\mathcal{V}, \mathcal{W}$ are $\Theta$-graded vector spaces:

$$
\mathcal{V}=\bigoplus_{i \in \Theta} v_{i}, \mathcal{W}=\bigoplus_{i \in \Theta} w_{i}
$$

Suppose there are linear maps $\pi_{i, j}: V_{i} \otimes V_{j} \rightarrow V_{i+j}$ and $\tau_{i, j}: W_{i} \otimes W_{j} \rightarrow W_{i+j}$ for every $i, j \in \Theta$. We refer to these maps as "products" on the vector spaces. Finally, suppose also there is an $\Theta$-graded isomorphism $\mathrm{T}: \mathcal{V} \rightarrow \mathcal{W}$ that preserves the products on $\mathcal{V}$ and $\mathcal{W}$ in the following sense: for all $i, j \in \Theta$ the following diagram commutes:

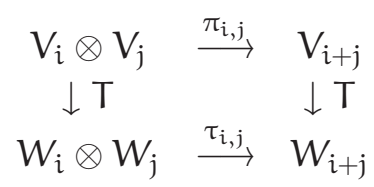

Then if $x \in V_{i}$ and $y \in V_{j}$ and $\tau_{i, j}(T(x) \otimes T(y)) \neq 0$, then $\pi_{i, j}(x \otimes y) \neq 0$.

For the purposes of the following lemma we will use the branching semigroup

$$
\Theta=\left\{(\gamma, \lambda) \in \Lambda_{n, n+1}: \gamma<\lambda\right\} .
$$

We introduce three $\Theta$-graded vector spaces, each of which is equipped with product maps.

The first space is $\mathcal{V}_{1}=\bigoplus_{(\gamma, \lambda) \in \Theta} \mathrm{V}^{\lambda}[\gamma]$. The product is defined as follows: for $x \in \mathrm{V}^{\lambda}[\gamma]$ and $x^{\prime} \in \mathrm{V}^{\lambda^{\prime}}\left[\gamma^{\prime}\right]$, define

$$
\chi \chi^{\prime}=p_{v+v^{\prime}}^{\lambda+\lambda^{\prime}}\left(\pi_{\lambda, \lambda^{\prime}}\left(\chi \otimes x^{\prime}\right)\right) .
$$


The second space is $\mathcal{V}_{2}=\bigoplus_{(\gamma, \lambda) \in \Theta} \mathrm{V}^{\gamma} \otimes \operatorname{Hom}_{\mathrm{GL}_{\mathrm{n}}}\left(\mathrm{V}^{\gamma}, \mathrm{V}^{\lambda}\right)$. The product is defined as follows: for $v \otimes f \in V^{\gamma} \otimes \operatorname{Hom}_{\mathrm{GL}_{n}}\left(\mathrm{~V}^{\gamma}, \mathrm{V}^{\lambda}\right)$ and $v^{\prime} \otimes \mathrm{f}^{\prime} \in \mathrm{V}^{\prime} \otimes \operatorname{Hom}_{\mathrm{GL}_{n}}\left(\mathrm{~V}^{\gamma^{\prime}}, \mathrm{V}^{\lambda^{\prime}}\right)$, define

$$
(v \otimes f)\left(v^{\prime} \otimes f^{\prime}\right)=\pi_{\gamma, \gamma^{\prime}}\left(v \otimes v^{\prime}\right) \otimes\left(\pi_{\lambda, \lambda^{\prime}} \circ\left(f \otimes f^{\prime}\right) \circ j_{\gamma, \gamma^{\prime}}\right) .
$$

The third space is $\mathcal{V}_{3}=\bigoplus_{(\gamma, \lambda) \in \Theta} \mathrm{V}^{\gamma} \otimes\left(\mathrm{V}^{\lambda}\right)^{\mathrm{u}_{\mathrm{n}}}(\gamma)$. The product is defined as follows: for $v \otimes w \in \mathrm{V}^{\gamma} \otimes\left(\mathrm{V}^{\lambda}\right)^{\mathrm{u}_{\mathrm{n}}}(\gamma)$ and $v^{\prime} \otimes w^{\prime} \in \mathrm{V}^{\gamma^{\prime}} \otimes\left(\mathrm{V}^{\lambda^{\prime}}\right)^{\mathrm{u}_{\mathrm{n}}}\left(\gamma^{\prime}\right)$, define

$$
(v \otimes w)\left(v^{\prime} \otimes w^{\prime}\right)=\pi_{\gamma, \gamma^{\prime}}\left(v \otimes v^{\prime}\right) \otimes \pi_{\lambda, \lambda^{\prime}}\left(w \otimes w^{\prime}\right) .
$$

Finally, we can state and prove the lemma. We note that it can also be obtained as a consequence of Theorem 1 in [Vin95]. We include an elementary proof for the sake of completeness.

Lemma 5.4. Let $(\nu, \lambda),\left(\nu^{\prime}, \lambda^{\prime}\right) \in \Lambda_{n, n+1}$. Suppose that $0 \neq x \in V^{\lambda}[v]$ and $0 \neq x^{\prime} \in$ $\mathrm{V}^{\lambda^{\prime}}\left[\boldsymbol{\nu}^{\prime}\right]$. Then $\mathrm{p}_{v+v^{\prime}}^{\lambda+\lambda^{\prime}}\left(\pi_{\lambda, \lambda^{\prime}}\left(\mathrm{x} \otimes \mathrm{x}^{\prime}\right)\right) \neq 0$.

Proof. Define $\mathrm{T}: \mathcal{V}_{2} \rightarrow \mathcal{V}_{1}$ by $\mathrm{T}(v \otimes f)=\mathrm{f}(v)$. This is clearly a linear isomorphism. Let $v \otimes f, v^{\prime} \otimes f^{\prime}$ be chosen as in the definition of $\mathcal{V}_{2}$. Then

$$
\mathrm{T}\left((\boldsymbol{v} \otimes \mathbf{f})\left(v^{\prime} \otimes \mathbf{f}^{\prime}\right)\right)=\left(\pi_{\lambda, \lambda^{\prime}} \circ\left(\mathrm{f} \otimes \mathbf{f}^{\prime}\right) \circ j_{\gamma, \gamma^{\prime}}\right)\left(\pi_{\gamma, \gamma^{\prime}}\left(v \otimes v^{\prime}\right)\right)
$$

while

$$
\mathrm{T}(v \otimes f) \mathrm{T}\left(v^{\prime} \otimes f^{\prime}\right)=p_{\gamma+\gamma^{\prime}}^{\lambda+\lambda^{\prime}}\left(\pi_{\lambda, \lambda^{\prime}}\left(f(v) \otimes f\left(v^{\prime}\right)\right)\right) .
$$

Let $z=v \otimes v^{\prime}$. Since $z \in V^{\gamma} \otimes V^{\gamma^{\prime}}$ we can write $z=z_{0}+z_{1}$, where $z_{0} \in\left(V^{\gamma} \otimes V^{\gamma^{\prime}}\right)\left[\gamma+\gamma^{\prime}\right]$ and $z_{1} \in \sum_{\tau \neq \gamma+\gamma^{\prime}}\left(\mathrm{V}^{\gamma} \otimes \mathrm{V}^{\prime}\right)[\tau]$. By the definition of $\pi_{\gamma, \gamma^{\prime}}$ and $j_{\gamma, \gamma^{\prime}}$, the composition $j_{\gamma, \gamma^{\prime}} \circ \pi_{\gamma, \gamma^{\prime}}$ is the projection of $V^{\gamma} \otimes V^{\gamma^{\prime}}$ onto its isotypic component $\left(V^{\gamma} \otimes V^{\gamma^{\prime}}\right)\left[\gamma+\gamma^{\prime}\right]$. Therefore,

$$
\left(\pi_{\lambda, \lambda^{\prime}} \circ\left(\mathbf{f} \otimes \mathbf{f}^{\prime}\right) \circ j_{\gamma, \gamma^{\prime}}\right)\left(\pi_{\gamma, \gamma^{\prime}}(z)\right)=\left(\pi_{\lambda, \lambda^{\prime}} \circ\left(\mathbf{f} \otimes f^{\prime}\right)\right)\left(z_{0}\right) .
$$

On the other hand,

$$
\begin{aligned}
p_{\gamma+\gamma^{\prime}}^{\lambda+\lambda^{\prime}}\left(\pi_{\lambda, \lambda^{\prime}}\left(f(v) \otimes f\left(v^{\prime}\right)\right)\right) & =p_{\gamma+\gamma^{\prime}}^{\lambda+\lambda^{\prime}}\left(\pi_{\lambda, \lambda^{\prime}}\left(\left(f \otimes f^{\prime}\right)\left(z_{0}\right)\right)\right)+p_{\gamma+\gamma^{\prime}}^{\lambda+\lambda^{\prime}}\left(\pi_{\lambda, \lambda^{\prime}}\left(\left(f \otimes f^{\prime}\right)\left(z_{1}\right)\right)\right) \\
& =\pi_{\lambda, \lambda^{\prime}}\left(\left(f \otimes f^{\prime}\right)\left(z_{0}\right)\right) .
\end{aligned}
$$

Therefore $T$ preserves the products on $\mathcal{V}_{1}$ and $\mathcal{V}_{2}$.

Next define $S: \mathcal{V}_{2} \rightarrow \mathcal{V}_{3}$ by $S(\nu \otimes f)=v \otimes f\left(\nu_{\gamma}\right)$ (recall that $v_{\gamma}$ is the canonical highest weight vector in $V^{\gamma}$ ). This is clearly a linear isomorphism. We show $S$ preserves the product maps. Let $v \otimes f, v^{\prime} \otimes f^{\prime}$ be chosen as in the definition of $\mathcal{V}_{2}$. Then

$$
\begin{aligned}
S\left((v \otimes f)\left(v^{\prime} \otimes f^{\prime}\right)\right) & =S\left(\pi_{\gamma, \gamma^{\prime}}\left(v \otimes v^{\prime}\right) \otimes\left(\pi_{\lambda, \lambda^{\prime}} \circ\left(f \otimes f^{\prime}\right) \circ j_{\gamma, \gamma^{\prime}}\right)\right) \\
& =\pi_{\gamma, \gamma^{\prime}}\left(v \otimes v^{\prime}\right) \otimes \pi_{\lambda, \lambda^{\prime}}\left(f\left(v_{\gamma}\right) \otimes f\left(v_{\gamma^{\prime}}\right)\right) \\
& =S(v \otimes f) S\left(v^{\prime} \otimes f^{\prime}\right) .
\end{aligned}
$$

Therefore $S$ preserves the products on $\mathcal{V}_{2}$ and $\mathcal{V}_{3}$.

Now $S \circ \mathrm{T}^{-1}$ is a graded isomorphism of $\mathcal{V}_{1}$ and $\mathcal{V}_{3}$ that respects products. Consider $0 \neq x \in V^{\lambda}[v]$. Under the isomorphism $S \circ T^{-1}, x$ is mapped to a simple tensor $v \otimes w$.

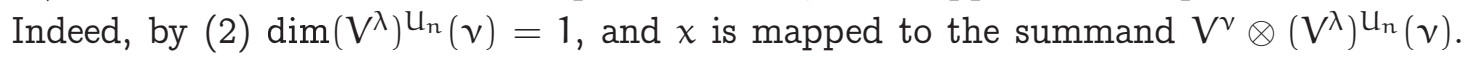


Similarly, $0 \neq x^{\prime} \in \mathrm{V}^{\lambda^{\prime}}\left[\mathrm{v}^{\prime}\right]$ is mapped to a simple tensor $v^{\prime} \otimes w^{\prime}$. By the definition of multiplication in $\mathcal{V}_{3}$, we have that $(v \otimes w)\left(v^{\prime} \otimes w^{\prime}\right)=\pi_{v, v^{\prime}}\left(v \otimes v^{\prime}\right) \otimes \pi_{\lambda, \lambda^{\prime}}\left(w \otimes w^{\prime}\right)$. Now, $\pi_{v, v^{\prime}}\left(v \otimes v^{\prime}\right)$ (resp. $\left.\pi_{\lambda, \lambda^{\prime}}\left(w \otimes w^{\prime}\right)\right)$ is simply the product of $v$ and $v^{\prime}$ (resp. $w$ and $\left.w^{\prime}\right)$ in $\mathcal{R}_{\mathrm{GL}_{n}}$ (resp. $\mathcal{R}_{\mathrm{GL}_{n+1}}$ ). Since $\mathcal{R}_{\mathrm{GL}_{n}}$ (resp. $\mathcal{R}_{\mathrm{GL}_{n+1}}$ ) has no zero divisors, it follows that $(v \otimes w)\left(v^{\prime} \otimes w^{\prime}\right) \neq 0$. By the observation above we conclude that $x x^{\prime} \neq 0$ in $\mathcal{V}_{1}$, i.e. $p_{\gamma+\gamma^{\prime}}^{\lambda+\lambda^{\prime}}\left(\pi_{\lambda, \lambda^{\prime}}\left(x \otimes x^{\prime}\right)\right) \neq 0$.

5.4. Proof of Proposition 3.6. Let $\left(t_{n}^{*}\right)_{\mathbb{R}}$ be the real form of $t_{n}^{*}$ spanned by $\left\{\varepsilon_{i}: i=\right.$ $1, \ldots, n\}$, where $\varepsilon_{i}$ is the functional mapping a diagonal matrix to its $i^{\text {th }}$ entry. Let $(\cdot, \cdot)$ be the inner product on $\left(t_{n}^{*}\right)_{\mathbb{R}}$ defined by $\left(\varepsilon_{i}, \varepsilon_{j}\right)=\delta_{i j}$, and let $\|\gamma\|^{2}=(\gamma, \gamma)$ define the associated norm. Denote by $\preceq$ the positive root ordering on $t_{n}^{*}$, defined relative to the set of positive roots: $\left\{\varepsilon_{i}-\varepsilon_{j}: i<j\right\}$. In other words, $\alpha \preceq \beta$ means $\beta-\alpha$ is a nonnegative integer combination of positive roots. Recall that for $\nu, \nu^{\prime}, \gamma \in \Lambda_{n}$, $\operatorname{Hom}_{\mathrm{GL}}\left(\mathrm{V}^{\gamma}, \mathrm{V}^{v} \otimes \mathrm{V}^{v^{\prime}}\right) \neq\{0\}$ implies $\gamma \preceq v+v^{\prime}$.

By Theorem 3.1, Proposition 3.6 is an immediate corollary of the following result:

Proposition 5.5. Let $\sigma \in \Sigma$ and let $(\mu, \lambda),\left(\mu^{\prime}, \lambda^{\prime}\right) \in \Omega(\sigma)$. Then the map

$$
V^{\lambda / \mu} \otimes V^{\lambda^{\prime} / \mu^{\prime}} \stackrel{\pi_{\lambda, \lambda^{\prime}}}{\longrightarrow} V^{\lambda+\lambda^{\prime} / \mu+\mu^{\prime}}
$$

is surjective.

Proof. To ease notation let $X=V^{\lambda / \mu}, X^{\prime}=V^{\lambda^{\prime} / \mu^{\prime}}, Y=V^{\lambda+\lambda^{\prime} / \mu+\mu^{\prime}}$, and $\pi=\pi_{\lambda, \lambda^{\prime}}$. For $\gamma \in \Lambda_{n}$ set $Y[\gamma]=p_{\gamma}^{\lambda+\lambda^{\prime}}(Y), X[\gamma]=p_{\gamma}^{\lambda}(X)$, and $X^{\prime}[\gamma]=p_{\gamma}^{\lambda^{\prime}}\left(X^{\prime}\right)$.

Note that $Y=\bigoplus_{\gamma \in \Lambda_{n}} Y[\gamma]$, and $\operatorname{dim} Y[\gamma]$ is zero or one. Moreover, $Y[\gamma] \neq\{0\}$ if, and only if, $\mu+\mu^{\prime}<\gamma<\lambda+\lambda^{\prime}$. We will prove by induction on $\|\gamma\|$ that $Y[\gamma]$ is in the image of $\pi$.

Let $\gamma \in \Lambda_{n}$ be of minimal norm such that $Y[\gamma] \neq\{0\}$. Our base case is to show that $Y[\gamma]$ is in the image of $\pi$. Since $(\mu, \lambda),\left(\mu^{\prime}, \lambda^{\prime}\right) \in \Omega(\sigma)$ we can apply Lemma 5.3 to obtain $v, v^{\prime} \in \Lambda_{n}$ such that $\gamma=v+v^{\prime}, \mu<v<\lambda$, and $\mu^{\prime}<v^{\prime}<\lambda^{\prime}$. Choose $0 \neq x \in X[v]$ and $0 \neq x^{\prime} \in X^{\prime}\left[v^{\prime}\right]$, and let $z=x \otimes x^{\prime}$.

Now, $\pi(z)=\sum_{\tau \in \Lambda_{n}} p_{\tau}^{\lambda+\lambda^{\prime}}(\pi(z))$ is a decomposition of $\pi(z)$ in $Y=\bigoplus_{\gamma \in \Lambda_{n}} Y[\gamma]$. Since $\mathrm{p}_{\tau}^{\lambda+\lambda^{\prime}}(\pi(z))=0$ for $\tau \succ \gamma, \pi(z)=\sum_{\tau \prec \gamma} \mathrm{p}_{\tau}^{\lambda+\lambda^{\prime}}(\pi(z))$. Now $\tau \prec \gamma$ implies $\|\tau\|<\|\gamma\|$, and by hypothesis $\gamma$ is of minimal norm such that $Y[\gamma] \neq\{0\}$. Therefore $p_{\tau}^{\lambda+\lambda^{\prime}}(\pi(z))=0$ for $\tau \prec \gamma$, and hence $\pi(z)=p_{\gamma}^{\lambda+\lambda^{\prime}}(\pi(z)) \in \mathrm{Y}[\gamma]$. By definition, $\pi(z)$ is the product of $x$ and $x^{\prime}$ in $\mathcal{R}_{\mathrm{GL}_{n+1}}$. Therefore, since $\mathcal{R}_{\mathrm{GL}_{n+1}}$ has no zero divisors, $\pi(z) \neq 0$. Since $\operatorname{dim} Y[\gamma]=1$, we conclude that $Y[\gamma]$ is in the image of $\pi$. This completes the base case.

Now fix $\gamma \in \Lambda_{n}$ such that $Y[\gamma] \neq\{0\}$, and suppose $Y[\tau]$ is in the image of $\pi$ for all $\tau$ such that $\|\tau\|<\|\gamma\|$. Using Lemma 5.3 again, we choose $\gamma, v^{\prime} \in \Lambda_{n-1}$ such that $\gamma=v+v^{\prime}, \mu<v<\lambda$, and $\mu^{\prime}<v^{\prime}<\lambda^{\prime}$. Also choose $0 \neq y \in Y[\gamma], 0 \neq x \in X[v]$, and $0 \neq x^{\prime} \in X^{\prime}\left[v^{\prime}\right]$. By Lemma 5.4, $p_{\gamma}^{\lambda+\lambda^{\prime}}\left(\pi\left(x \otimes x^{\prime}\right)\right) \neq 0$. Therefore

$$
\pi\left(x \otimes x^{\prime}\right) \in \mathbb{C}^{\times} y+\sum_{\tau \prec \gamma} Y[\tau] .
$$


Since $\tau \prec \gamma$ implies $\|\tau\|<\|\gamma\|$, by the inductive hypothesis we obtain an element $\xi \in X \otimes X^{\prime}$ such that $\pi(\xi)=y$. Since $\operatorname{dim} Y[\gamma]=1$, this shows that $Y[\gamma]$ is in the image of $\pi$. This completes the induction.

\section{ProOF OF THEOREM 3.5}

In this section it will be convenient for us to introduce the following convention. Elements of the branching semigroup $\Lambda_{\mathcal{B}}$ will be thought of as "skew shapes", and so instead of writing $(\mu, \lambda) \in \Lambda_{\mathcal{B}}$, we will write $\lambda / \mu \in \Lambda_{\mathcal{B}}$. In this way, for $p=\lambda / \mu \in \Lambda_{\mathcal{B}}$ we associate the spaces $W^{p}, A^{p}$, etc...

6.1. A filtration on the branching semigroup. Let $h: \Lambda_{\mathcal{B}} \rightarrow \Lambda_{2 n}$ be given by $h(\lambda / \mu)=f\left(\mu, \lambda^{+}\right)$, where $f\left(\mu, \lambda^{+}\right)$is the rearrangement function defined in Section 5.1 . The image of $h$ is thus all sequences in $\Lambda_{2 n}$ ending in zero. As before, we define the functions $r_{i}: \Lambda_{\mathcal{B}} \rightarrow \mathbb{Z}$ by $r_{i}(p)=x_{i}-y_{i}$, where $h(p)=\left(x_{1}, y_{1}, \ldots, x_{n}, y_{n}\right)$. Moreover, for $\sigma \in \Sigma$, let $h_{\sigma}$ denote the restriction of $h$ to $\Lambda_{\mathcal{B}}(\sigma)$.

The same argument as in Lemma 5.2 shows:

Lemma 6.1. Let $\sigma \in \Sigma$. Then $h_{\sigma}: \Lambda_{\mathcal{B}}(\sigma) \rightarrow \Lambda_{2 n}$ is a semigroup embedding, with image the sequences in $\Lambda_{2 n}$ ending in zero. In particular, $h_{\sigma}^{-1}$ is defined on the set of such sequences.

In this section we will only deal with sequences ending in zero, so $h_{\sigma}^{-1}$ will always be well-defined. By the above lemma we endow the L-module

$$
\mathcal{A}_{\sigma}=\bigoplus_{p \in \wedge_{\mathcal{B}}(\sigma)} A^{p}
$$

with a product given by Cartan product of irreducible L-modules: $A^{p} \otimes A^{p^{\prime}} \rightarrow A^{p+p}$.

We now show that $\mathcal{A}_{\sigma}$ is a very naturally occurring L-algebra. Consider the L-module $\mathrm{V}=\mathrm{U} \times \mathrm{W}$, where $\mathrm{U}=\mathbb{C}^{2} \times \cdots \times \mathbb{C}^{2}$ ( $\mathrm{n}$ copies $)$ and $\mathrm{W}=\mathbb{C} \times \cdots \times \mathbb{C}(\mathrm{n}-1$ copies $)$. Here, $\mathrm{L}$ acts on $\mathrm{U}$ diagonally, on $\mathrm{W}$ trivially, and on the ring of functions $\mathcal{O}(\mathrm{V})$ by right translation. Let $t_{1}, \ldots, t_{n-1}$ be the standard coordinate functions on $\mathbb{C}^{n-1}$. Decompose $\mathcal{O}(\mathrm{V})$ into graded components:

$$
\mathcal{O}(V) \cong \bigoplus_{\substack{r_{j} \geq 0 \\ j=1, \ldots, n}} \bigoplus_{\substack{s_{k} \geq 0 \\ k=1, \ldots, n-1}} F^{r_{1}} \otimes \cdots \otimes F^{r_{n}} \otimes t_{1}^{s_{1}} \cdots t_{n-1}^{s_{n-1}} .
$$

This is also a decomposition of $\mathcal{O}(\mathrm{V})$ into irreducible L-modules.

For $\sigma \in \Sigma$, we can consider $\mathcal{O}(\mathrm{V})$ as an $\Lambda_{\mathcal{B}}(\sigma)$-graded algebra as follows. Set $s_{i}$ : $\Lambda_{\mathcal{B}}(\sigma) \rightarrow \mathbb{Z}$ by $s_{i}(p)=y_{i}-x_{i+1}$, where, as usual, $h(p)=\left(x_{1}, y_{1}, \ldots, x_{n}, y_{n}\right)$. Then define the p-component of $\mathcal{O}(\mathrm{V})$ by:

$$
\mathcal{O}(V)^{p}=F^{r_{1}(p)} \otimes \cdots \otimes F^{r_{n}(p)} \otimes t_{1}^{s_{1}(p)} \cdots t_{n-1}^{s_{n-1}(p)}
$$

Clearly, we have

$$
\mathcal{O}(\mathrm{V})=\bigoplus_{\mathrm{p} \in \Lambda_{\mathcal{B}}(\sigma)} \mathcal{O}(\mathrm{V})^{\mathrm{p}}
$$

One easily proves the following lemma. 
Lemma 6.2. Let $\sigma \in \Sigma$ and regard $\mathcal{O}(\mathrm{V})$ as an $\Lambda_{\mathcal{B}}(\sigma)$-graded L-algebra. Then $\mathcal{A}_{\sigma}$ and $\mathcal{O}(\mathrm{V})$ are isomorphic as $\Lambda_{\mathcal{B}}(\sigma)$-graded L-algebras, and the isomorphism is unique up to scalars.

The main step in proving Theorem 3.5 is showing that $\mathcal{A}_{\sigma}$ and $\mathcal{B}_{\sigma}$ are isomorphic as $\mathrm{SL}_{2}$-algebras, and the isomorphism is unique up to scalars. We will prove this by induction on a certain filtration of $\Lambda_{\mathcal{B}}(\sigma)$, which we now describe.

For $p \in \Lambda_{\mathcal{B}}$ let $p_{\max }=\lambda_{1}$ where $p=\lambda / \mu$ and $\lambda=\left(\lambda_{1}, \ldots, \lambda_{n}\right)$. For every $\sigma \in \Sigma$ we define the set

$$
\Lambda_{\mathcal{B}}(\sigma, m)=\left\{p \in \Lambda_{\mathcal{B}}(\sigma): p_{\max } \leq \mathrm{m}\right\}
$$

Clearly $\Lambda_{\mathcal{B}}(\sigma, m)$ is finite, $\Lambda_{\mathcal{B}}(\sigma, m-1) \subset \Lambda_{\mathcal{B}}(\sigma, m)$, and $\bigcup_{m \geq 0} \Lambda_{\mathcal{B}}(\sigma, m)=\Lambda_{\mathcal{B}}(\sigma)$.

Lemma 6.3. Let $m>1, \sigma \in \Sigma$, and suppose $p \in \Lambda_{\mathcal{B}}(\sigma, m)$ satisfies $p_{\max }=m$.

(1) There exist $p^{\prime}, p^{\prime \prime} \in \Lambda_{\mathcal{B}}(\sigma, m-1)$ such that $p=p^{\prime}+p^{\prime \prime}$.

(2) Suppose moreover that $\tau \in \Sigma$ and $p \in \Lambda_{\mathcal{B}}(\tau, \mathrm{m})$. Then there exist $\mathrm{p}^{\prime}, \mathrm{p}^{\prime \prime} \in$ $\Lambda_{\mathcal{B}}(\sigma, m-1) \cap \Lambda_{\mathcal{B}}(\tau, m-1)$ such that $p=p^{\prime}+p^{\prime \prime}$.

Proof. Let $h_{\sigma}(p)=\left(z_{1}, \ldots, z_{2 n}\right)$. Define

$$
z_{i}^{\prime}=\left\{\begin{array}{l}
1 \text { if } z_{i} \geq 1 \\
0 \text { if } z_{i}=0
\end{array}\right.
$$

and $z_{i}^{\prime \prime}=z_{i}-z_{i}^{\prime}$. It's trivial to check that $\xi^{\prime}=\left(z_{1}^{\prime}, \ldots, z_{2 n}^{\prime}\right), \xi^{\prime \prime}=\left(z_{1}^{\prime \prime}, \ldots, z_{2 n}^{\prime \prime}\right) \in \Lambda_{2 n}$. Let $p^{\prime}=f_{\sigma}^{-1}\left(\xi^{\prime}\right)$ and $p^{\prime \prime}=f_{\sigma}^{-1}\left(\xi^{\prime \prime}\right)$. This is well-defined by Lemma 6.1. Lemma 6.1 also shows that $p=p^{\prime}+p^{\prime \prime}$. Since $m>1, p^{\prime}, p^{\prime \prime} \in \Lambda_{\mathcal{B}}(\sigma, m-1)$. This proves (1).

Let $\mathrm{p}^{\prime}, \mathrm{p}^{\prime \prime} \in \Lambda_{\mathcal{B}}(\sigma, \mathrm{m}-1)$ be constructed as in the previous paragraph. We must show that $p^{\prime}, p^{\prime \prime} \in \Lambda_{\mathcal{B}}(\sigma) \cap \Lambda_{\mathcal{B}}(\tau)$. If $\sigma=\left(\sigma_{1} \cdots \sigma_{n-1}\right)$ and $\tau=\left(\tau_{1} \cdots \tau_{n-1}\right)$, then every $i$ such that $\sigma_{i} \neq \tau_{i}$ forces the equality $\mu_{i}=\lambda_{i+1}$ among the entries of $p$. Therefore, if $h_{\sigma}(p)=\left(z_{1}, \ldots, z_{2 n}\right)$ and $\sigma_{i} \neq \tau_{i}$, then $z_{2 i+1}=z_{2 i+2}$. Now note that in the definition of $\xi^{\prime}, \xi^{\prime \prime}$, if $z_{2 i+1}=z_{2 i+2}$ then $z_{2 i+1}^{\prime}=z_{2 i+2}^{\prime}$ and $z_{2 i+1}^{\prime \prime}=z_{2 i+2}^{\prime \prime}$. Hence the entries of $\xi^{\prime}, \xi^{\prime \prime}$ satisfy the same equalities that $h_{\sigma}(p)$ satisfies, which implies that $p^{\prime}, p^{\prime \prime} \in \Lambda_{\mathcal{B}}(\sigma) \cap \Lambda_{\mathcal{B}}(\tau)$. This proves (2).

Lemma 6.4. Let $m>1, \sigma \in \Sigma$, and suppose $p \in \Lambda_{\mathcal{B}}(\sigma, m)$ satisfies $p_{\max }=m$. Then there exist $\mathrm{q}_{1}, \ldots, \mathrm{q}_{\mathrm{n}} \in \Lambda_{\mathcal{B}}(\sigma, \mathrm{m}-1)$ such that

(1) $\mathrm{p}=\mathrm{q}_{1}+\cdots+\mathrm{q}_{\mathrm{n}}$

(2) $A^{q_{i}}$ is an irreducible $\mathrm{SL}_{2}$-module.

(3) Either $A^{q_{1}} \otimes \cdots \otimes A^{q_{n}} \cong A^{p}$ as $S_{2}$-modules, or $A^{p}$ is irreducible as an $\mathrm{SL}_{2}$ module, and the multiplication map $A^{q_{1}} \otimes \cdots \otimes A^{q_{n}} \rightarrow A^{p}$ is a projection onto the Cartan component $A^{p}$ of $A^{q_{1}} \otimes \cdots \otimes A^{q_{n}}$.

Proof. Let $h_{\sigma}(p)=\left(x_{1}, y_{1}, \ldots, x_{n}, y_{n}\right)$. Define

$$
\xi_{i}=(\underbrace{x_{i}-x_{i+1}, \ldots, x_{i}-x_{i+1}}_{2 i-1}, y_{i}-x_{i+1}, 0, \ldots, 0)
$$

for $i=1, \ldots, n-1$, and set $\xi_{n}=\left(x_{n}, \ldots, x_{n}, 0\right)$. The argument breaks into cases. 
Case 1: Suppose $h_{\sigma}^{-1}\left(\xi_{i}\right) \notin \Lambda_{\mathcal{B}}(\sigma, m-1)$ for some $i \leq n$. Then $x_{i}-x_{i+1}=m$ and therefore

$$
h_{\sigma}(p)=(m, \ldots, m, b, 0, \ldots, 0)
$$

for some $b \leq m$ in the $(2 i)^{\text {th }}$ entry. Therefore $A^{p}$ is irreducible as an $S_{2}$-module.

Now choose $\xi^{\prime}, \xi^{\prime \prime}$ as in the proof of Lemma 6.3 and consider the associated $p^{\prime}, p^{\prime \prime}$. By the lemma $p^{\prime}, p^{\prime \prime} \in \Lambda_{\mathcal{B}}(\sigma, m-1)$. Moreover, by our construction of $\xi^{\prime}, \xi^{\prime \prime}$ from $\xi, \mathcal{A}^{\mathrm{p}^{\prime}}, \mathcal{A}^{\mathrm{p}^{\prime \prime}}$ are irreducible $\mathrm{SL}_{2}$-modules. Therefore the map $A^{\mathrm{p}^{\prime}} \otimes A^{\mathrm{p}^{\prime \prime}} \rightarrow A^{\mathrm{p}}$ is a projection onto the Cartan component of $A^{p^{\prime}} \otimes A^{p^{\prime \prime}}$, and the lemma is satisfied with $q_{1}=p^{\prime}, q_{2}=p^{\prime \prime}$, and $q_{i}=0$ for $i>2$.

Case 2: Suppose that $h_{\sigma}^{-1}\left(\xi_{i}\right) \in \Lambda_{\mathcal{B}}(\sigma, m-1)$ for $i=1, \ldots, n$. Then set $q_{i}=h_{\sigma}^{-1}\left(\xi_{i}\right)$. Since $\xi=\xi_{1}+\cdots+\xi_{n}$, by Lemma [5.2, $p=q_{1}+\cdots+q_{n}$. By the definition of $\xi_{i}$ we also have that

$$
A^{q_{i}}=F^{0} \otimes \cdots \otimes \underbrace{F^{x_{i}-y_{i}}}_{i^{\text {th }}} \otimes \cdots \otimes F^{0} .
$$

Therefore $A^{q_{i}}$ is an irreducible $S L_{2}$-module, and $A^{q_{1}} \otimes \cdots \otimes A^{q_{n}} \cong A^{p}$.

Remark 6.5. In the proof of Lemma 6.4 all we used was the $\mathrm{SL}_{2}$-module structure of $A^{p}$. Therefore, by Corollary [3.3, the statement holds with $A^{p}$ replaced by $W^{p}$ and $A^{q_{i}}$ replaced by $W^{q_{i}}$.

Lemma 6.6. Let $\mathrm{m}>1, \sigma \in \Sigma$, and suppose $p \in \Lambda_{\mathcal{B}}(\sigma, \mathrm{m})$ satisfies $\mathrm{p}_{\max }=\mathrm{m}$. Let $\mathrm{q}_{1}, \ldots, \mathrm{q}_{\mathrm{n}} \in \Lambda_{\mathcal{B}}(\sigma, \mathrm{m}-1)$ be given as in Lemma 6.4. Suppose also we are given $\mathrm{SL}_{2}$-isomorphisms $\phi_{i}: W^{q_{i}} \rightarrow A^{q_{i}}$ for $i=1, \ldots, n$. Let

$$
\begin{aligned}
K & =\operatorname{ker}\left(W^{q_{1}} \otimes \cdots \otimes W^{q_{n}} \stackrel{\tau}{\rightarrow} W^{p}\right) \\
J & =\operatorname{ker}\left(A^{q_{1}} \otimes \cdots \otimes A^{q_{n}} \stackrel{\kappa}{\rightarrow} A^{p}\right)
\end{aligned}
$$

be the kernels of the multiplication maps coming from the rings $\mathcal{B}_{\sigma}$ and $\mathcal{A}_{\sigma}$, which we denote here by $\tau$ and $\mathrm{K}$. Set $\phi=\phi_{1} \otimes \cdots \otimes \phi_{n}$. Then $\phi(\mathrm{K})=\mathrm{J}$. Consequently, there is an $\mathrm{SL}_{2}$-isomorphism $\psi: W^{p} \rightarrow A^{p}$ making the following diagram commute:

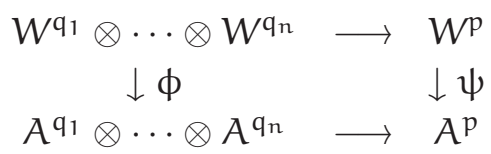

Proof. Clearly $\mathrm{k}$ is surjective. By Proposition 3.6, $\tau$ is surjective. Therefore we have the following diagram:

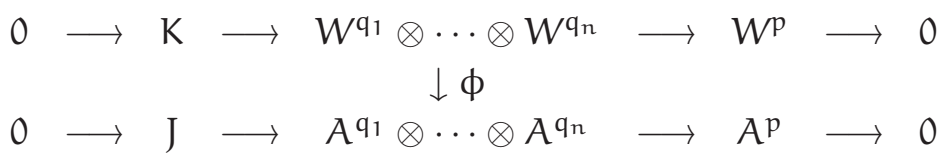

According to Lemma 6.4 there are two possibilities. Either $A^{q_{1}} \otimes \cdots \otimes A^{q_{n}} \cong A^{p}$ as $\mathrm{SL}_{2}$-modules, or $A^{p}$ is irreducible as a $\mathrm{SL}_{2}$-module, and the multiplication map $A^{q_{1}} \otimes$ $\cdots \otimes A^{q_{n}} \rightarrow A^{p}$ is a projection onto the Cartan component $A^{p}$ of $A^{q_{1}} \otimes \cdots \otimes A^{q_{n}}$. If $A^{q_{1}} \otimes \cdots \otimes A^{q_{n}} \cong A^{p}$ then $J=\{0\}$, and by the above remark $W^{q_{1}} \otimes \cdots \otimes W^{q_{n}} \cong W^{p}$. Therefore $\mathrm{K}=\{0\}$, and so clearly $\phi(\mathrm{K})=\mathrm{J}$. 
In the other case, $A^{p} \cong W^{p}$ are irreducible $S L_{2}$-modules. Choose $k$ so that $A^{p} \cong$ $W^{p} \cong F^{k}$. Since the maps $\kappa, \tau$ are both projections onto the Cartan component $F^{k}$, their kernels are given as sums of $\mathrm{SL}_{2}$-isotypic components

$$
\begin{aligned}
K & =\sum_{n<k}\left(W^{q_{1}} \otimes \cdots \otimes W^{q_{n}}\right)[j] \\
J & =\sum_{j<k}\left(A^{q_{1}} \otimes \cdots \otimes A^{q_{n}}\right)[j] .
\end{aligned}
$$

Since $\phi$ intertwines the $\mathrm{SL}_{2}$-action, $\phi(\mathrm{K}) \subset \mathrm{J}$. Moreover, $\mathrm{K}$ and $\tau$ are both Cartan multiplications of the same $\mathrm{SL}_{2}$-modules, and so $\operatorname{dim} \mathrm{K}=\operatorname{dim} \mathrm{J}$. Therefore $\phi(\mathrm{K})=\mathrm{J}$.

Lemma 6.7. Let $m>1, \sigma \in \Sigma$, and suppose we are given $\mathrm{p}^{\prime}, \mathrm{p}^{\prime \prime}, \mathrm{q}^{\prime}, \mathrm{q}^{\prime \prime} \in \Lambda_{\mathcal{B}}(\sigma, \mathrm{m})$ such that $\mathrm{p}^{\prime}+\mathrm{p}^{\prime \prime}=\mathrm{q}^{\prime}+\mathrm{q}^{\prime \prime}$. Then there exist $\mathrm{t}^{\prime}, \mathrm{t}^{\prime \prime}, \mathrm{r}^{\prime}, \mathrm{r}^{\prime \prime} \in \Lambda_{\mathcal{B}}(\sigma, \mathrm{m})$ such that

$$
\begin{aligned}
& \mathrm{t}^{\prime}+\mathrm{r}^{\prime}=\mathrm{p}^{\prime}, \mathrm{t}^{\prime \prime}+\mathrm{r}^{\prime \prime}=\mathrm{p}^{\prime \prime} \\
& \mathrm{t}^{\prime}+\mathrm{t}^{\prime \prime}=\mathrm{q}^{\prime}, \mathrm{r}^{\prime}+\mathrm{r}^{\prime \prime}=\mathrm{q}^{\prime \prime}
\end{aligned}
$$

Proof. For some nonnegative integers $n_{i}^{\prime}, n_{i}^{\prime \prime}, m_{i}^{\prime}, m_{i}^{\prime \prime}$ we have

$$
\begin{aligned}
& h_{\sigma}\left(p^{\prime}\right)=\sum_{i=1}^{2 n} n_{i}^{\prime} \varpi_{i}, h_{\sigma}\left(p^{\prime \prime}\right)=\sum_{i=1}^{2 n} n_{i}^{\prime \prime} \varpi_{i} \\
& h_{\sigma}\left(q^{\prime}\right)=\sum_{i=1}^{2 n} m_{i}^{\prime} \varpi_{i}, h_{\sigma}\left(q^{\prime \prime}\right)=\sum_{i=1}^{2 n} m_{i}^{\prime \prime} \varpi_{i}
\end{aligned}
$$

Define:

$$
\begin{aligned}
\tau^{\prime} & =\sum_{i=1}^{2 n}\left(m_{i}^{\prime \prime}-\min \left(n_{i}^{\prime \prime}, m_{i}^{\prime \prime}\right)\right) \varpi_{i} \\
\rho^{\prime} & =\sum_{i=1}^{2 n}\left(n_{i}^{\prime}-m_{i}^{\prime \prime}+\min \left(n_{i}^{\prime \prime}, m_{i}^{\prime \prime}\right)\right) \varpi_{i} \\
\tau^{\prime \prime} & =\sum_{i=1}^{2 n} \min \left(n_{i}^{\prime \prime}, m_{i}^{\prime \prime}\right) \varpi_{i} \\
\rho^{\prime \prime} & =\sum_{i=1}^{2 n}\left(n_{i}^{\prime \prime}-\min \left(n_{i}^{\prime \prime}, m_{i}^{\prime \prime}\right)\right) \varpi_{i} .
\end{aligned}
$$

Clearly $\tau^{\prime}, \tau^{\prime \prime}, \rho^{\prime \prime} \in \Lambda_{2 n}$. Since $p^{\prime}+p^{\prime \prime}=q^{\prime}+q^{\prime \prime}, \rho^{\prime} \in \Lambda_{2 n}$ as well. Now, note that

$$
\begin{gathered}
h_{\sigma}\left(p^{\prime}\right)=\tau^{\prime}+\rho^{\prime}, h_{\sigma}\left(p^{\prime \prime}\right)=\tau^{\prime \prime}+\rho^{\prime \prime} \\
h_{\sigma}\left(q^{\prime}\right)=\rho^{\prime}+\rho^{\prime \prime}, h_{\sigma}\left(q^{\prime \prime}\right)=\tau^{\prime}+\tau^{\prime \prime} .
\end{gathered}
$$

Set

$$
\begin{aligned}
& t^{\prime}=h_{\sigma}^{-1}\left(\tau^{\prime}\right), \quad t^{\prime \prime}=h_{\sigma}^{-1}\left(\tau^{\prime \prime}\right) \\
& r^{\prime}=h_{\sigma}^{-1}\left(\rho^{\prime}\right), \quad r^{\prime \prime}=h_{\sigma}^{-1}\left(\rho^{\prime \prime}\right)
\end{aligned}
$$

Since $h_{\sigma}$ is a semigroup isomorphism and $p^{\prime}, p^{\prime \prime}, q^{\prime}, q^{\prime \prime} \in \Lambda_{\mathcal{B}}(\sigma)$, it follows that $t^{\prime}, t^{\prime \prime}, r^{\prime}, r^{\prime \prime} \in$ $\Lambda_{\mathcal{B}}(\sigma)$ and they satisfy the desired equations. 


\subsection{Proof of Proposition 3.7.}

Definition 6.8. Let $\mathcal{F}=\left\{\phi_{\mathrm{p}}: \mathrm{W}^{\mathrm{p}} \rightarrow \mathrm{A}^{\mathrm{p}}\right\}_{\mathrm{p} \in \Lambda_{\mathcal{B}}}$ be a family of $\mathrm{SL}_{2}$-isomorphisms indexed by $\Lambda_{\mathcal{B}}$. Then $\mathcal{F}$ is a compatible family if for any $\sigma \in \Sigma$ and $p^{\prime}, p^{\prime \prime} \in \Lambda_{\mathcal{B}}(\sigma)$ the following diagram commutes:

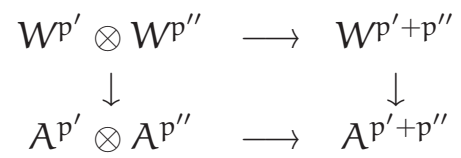

Here the vertical maps are given by $\phi_{p^{\prime}} \otimes \phi_{p^{\prime \prime}}$ and $\phi_{p^{\prime}+p^{\prime \prime}}$, and the horizontal maps are the product maps in the rings $\mathcal{B}_{\sigma}$ and $\mathcal{A}_{\sigma}$.

Proposition 6.9. There exists a compatible family $\mathcal{F}=\left\{\phi_{\mathfrak{p}}: W^{p} \rightarrow A^{p}\right\}_{\mathfrak{p} \in \Lambda_{\mathcal{B}}}$ of $\mathrm{SL}_{2}$-isomorphisms. Moreover, each map $\phi_{\mathrm{p}} \in \mathcal{F}$ is unique up to scalar.

Proof. For a nonnegative integer $m$ set $\Lambda_{\mathcal{B}}(m)=\left\{p \in \Lambda_{\mathcal{B}}: p_{\max } \leq m\right\}$. We first prove by induction on $m$ that there is a family of $S L_{2}$ isomorphisms

$$
\mathcal{F}_{\mathrm{m}}=\left\{\phi_{\mathrm{p}}: \mathrm{W}^{\mathrm{p}} \rightarrow \mathrm{A}^{\mathrm{p}}\right\}_{\mathrm{p} \in \Lambda_{\mathcal{B}}(\mathrm{m})}
$$

such that for any $p \in \Lambda_{\mathcal{B}}(m), \sigma \in \Sigma$, and $p^{\prime}, p^{\prime \prime} \in \Lambda_{\mathcal{B}}(\sigma)$ such that $p=p^{\prime}+p^{\prime \prime}$, diagram (16) commutes.

For the base case we construct $\mathcal{F}_{1}$. If $p_{\max }=0$ then $p=p_{0}=0 / 0$. We define $\phi_{p_{0}}: W^{p_{0}} \rightarrow A^{p_{0}}$ by $1 \in W^{p_{0}} \mapsto 1 \otimes \cdots \otimes 1 \in A^{p_{0}}$. Of course, if $p^{\prime}+p^{\prime \prime}=p_{0}$ then $p^{\prime}=p^{\prime \prime}=p_{0}$ and (16) trivially commutes. Suppose now that $p_{\max }=1$. Then $p=\lambda / \mu$ with $\lambda$ a fundamental weight, and $\mu$ either zero or a fundamental weight. In any case, $A^{p}$ is an irreducible $S L_{2}$-module, and by Corollary 3.3, $W^{p} \cong A^{p}$ as $S_{2}$-modules. We choose an $\mathrm{SL}_{2}$-isomorphism $\phi_{p}: W^{p} \rightarrow A^{p}$. By Schur's Lemma, $\phi_{p}$ is unique up to scalar. Now suppose $\sigma \in \Sigma, p^{\prime}, p^{\prime \prime} \in \Lambda_{\mathcal{B}}(\sigma)$, and $p^{\prime}+p^{\prime \prime}=p$. Then either $p_{\max }^{\prime}=0$ or $p_{\max }^{\prime \prime}=0$. Assume, without loss of generality, that $p_{\max }^{\prime}=0$. Then $p^{\prime}=p_{0}$, and by our construction of $\phi_{p_{0}}$, diagram (16) commutes. Set $\mathcal{F}_{1}=\left\{\phi_{p}: W^{p} \rightarrow A^{p}\right\}_{p \in \Lambda_{\mathcal{B}}(1)}$; this completes the base case.

Let $m>1$ and suppose that $\mathcal{F}_{m-1}$ exists and satisfies the desired properties. We must construct $\mathcal{F}_{\mathrm{m}}$. For $\mathrm{p} \in \Lambda_{\mathcal{B}}(\mathrm{m})$ such that $p_{\max }<\mathrm{m}$, there exists $\phi_{\mathrm{p}} \in \mathcal{F}_{\mathrm{m}-1}$ by hypothesis. We include these $\phi_{p}$ in $\mathcal{F}_{m}$. For such $p$ we have the following: if $\sigma \in \Sigma$, $p^{\prime}, p^{\prime \prime} \in \Lambda_{\mathcal{B}}(\sigma)$, and $p=p^{\prime}+p^{\prime \prime}$, then diagram (16) commutes. Indeed, $p=p^{\prime}+p^{\prime \prime}$ implies that $p^{\prime}, p^{\prime \prime} \in \Lambda_{\mathcal{B}}(m-1)$. Therefore $\phi_{p^{\prime}}$ and $\phi_{p^{\prime \prime}}$ are also obtained from $\mathcal{F}_{m-1}$, and diagram (16) commutes by hypothesis.

Suppose $p \in \Lambda_{\mathcal{B}}(\mathrm{m})$ satisfies $p_{\max }=m$. Choose an order type $\sigma \in \Sigma$ such that $p \in \Lambda_{B}(\sigma)$. Note that $\sigma$ may not be unique. Choose $q_{1}, \ldots, q_{n} \in \Lambda_{\mathcal{B}}(\sigma, m-1)$ by Lemma 6.4. Now apply Lemma 6.6 to obtain an $S_{2}$-isomorphism $\psi: W^{p} \rightarrow A^{p}$ such that the following diagram commutes:

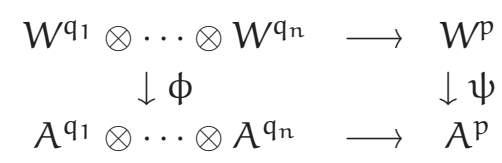


where $\phi=\phi_{\mathrm{q}_{1}} \otimes \cdots \otimes \phi_{\mathrm{q}_{\mathrm{n}}}$. We now show that (i) if $\mathrm{p}^{\prime}, \mathrm{p}^{\prime \prime} \in \Lambda_{\mathcal{B}}(\sigma)$ satisfy $\mathrm{p}=\mathrm{p}^{\prime}+\mathrm{p}^{\prime \prime}$ then

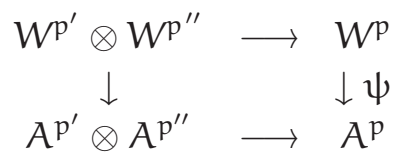

commutes, (ii) $\psi$ is independent of the choice of $q_{1}, \ldots, q_{n}$, and (iii) $\psi$ is independent of the choice of $\sigma$.

First note that (i) implies (ii). Indeed, suppose $q_{1}^{\prime}, \ldots, q_{n}^{\prime} \in \Lambda_{\mathcal{B}}(\sigma, m-1)$ is another collection of shapes satisfying the conditions of Lemma 6.4 and $\psi^{\prime}: W^{p} \rightarrow A^{p}$ is the associated $\mathrm{SL}_{2}$-isomorphism obtained by Lemma 6.6. By (i) both $\psi$ and $\psi^{\prime}$ would make (18) commute. But since all the maps in the diagram are surjective, there is a unique map making (18) commute. Therefore $\psi=\psi^{\prime}$.

Now we prove (i). If $p_{\max }^{\prime}=m\left(\right.$ resp. $\left.p_{\max }^{\prime \prime}=m\right)$ then $p^{\prime \prime}=p_{0}\left(\right.$ resp. $\left.p^{\prime}=p_{0}\right)$, and (18) commutes by our choice of $\phi_{p_{0}}$. Therefore we may assume that $p_{\max }^{\prime}, p_{\max }^{\prime \prime}<m$. By renumbering the $q_{j}$ if necessary, we may assume that $\left(q_{1}\right)_{\max } \neq 0$. Let $q^{\prime}=q_{1}$ and $\mathrm{q}^{\prime \prime}=\mathrm{q}_{2}+\cdots+\mathrm{q}_{\mathrm{n}}$. Then $\mathrm{q}^{\prime}, \mathrm{q}^{\prime \prime} \in \Lambda_{\mathcal{B}}(\sigma, \mathrm{m}-1)$ and $\mathrm{q}^{\prime}+\mathrm{q}^{\prime \prime}=\mathrm{p}$. By inductive hypothesis the following diagram commutes:

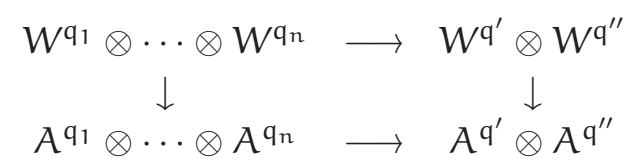

where the vertical map on the left is $\phi=\phi_{\mathrm{q}_{1}} \otimes \cdots \otimes \phi_{\mathrm{q}_{\mathrm{n}}}$, and the one on the right is $\phi_{\mathrm{q}^{\prime}} \otimes \phi_{\mathrm{q}^{\prime \prime}}$. Combining (17) and (19) and the fact that all the maps are surjective (Proposition 3.6), we conclude that

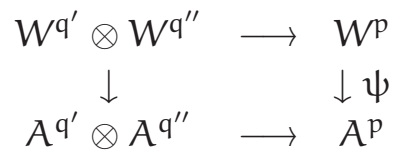

commutes.

Since $\mathrm{p}^{\prime}+\mathrm{p}^{\prime \prime}=\mathrm{q}^{\prime}+\mathrm{q}^{\prime \prime}$, by Lemma 6.7 there exist $\mathrm{t}^{\prime}, \mathrm{t}^{\prime \prime}, \mathrm{r}^{\prime}, \mathrm{r}^{\prime \prime} \in \Lambda_{\mathcal{B}}(\sigma, \mathrm{m})$ such that

$$
\begin{aligned}
t^{\prime}+r^{\prime} & =p^{\prime}, t^{\prime \prime}+r^{\prime \prime}=p^{\prime \prime} \\
t^{\prime}+t^{\prime \prime} & =q^{\prime}, r^{\prime}+r^{\prime \prime}=q^{\prime \prime} .
\end{aligned}
$$


Consider the following diagram:

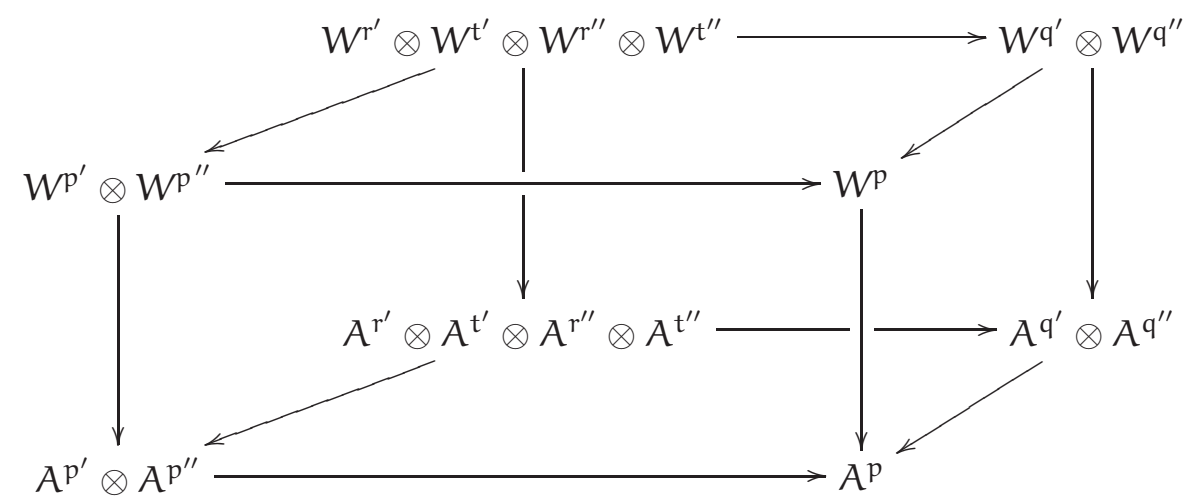

The top square commutes by associativity of the product in $\mathcal{B}_{\sigma}$. The left and back squares commute by inductive hypothesis. The right square commutes since it is the diagram (20). The bottom square commutes by associativity of the product in $\mathcal{A}_{\sigma}$. By chasing this diagram and repeatedly using Proposition 3.6, it follows that the front square commutes. This proves (ii).

We now prove (iii), namely that $\psi$ is independent of $\sigma$. Indeed, suppose $\tau \in \Sigma$ is another order type such that $p \in \Lambda_{\mathcal{B}}(\tau)$. By the above argument we obtain an $S L_{2}$ isomorphism $\zeta: W^{p} \rightarrow A^{p}$ such that (18) commutes for all $p^{\prime}, p^{\prime \prime} \in \Lambda_{\mathcal{B}}(\tau)$. By Lemma 6.3(2) there exist $p^{\prime}, p^{\prime \prime} \in \Lambda_{\mathcal{B}}(\sigma) \cap \Lambda_{\mathcal{B}}(\tau)$ such that $p=p^{\prime}+p^{\prime \prime}$. Therefore both $\psi$ and $\zeta$ make the following diagram commute:

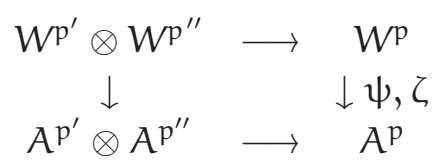

Hence $\psi=\zeta$.

At this point we've shown for any $p \in \Lambda_{\mathcal{B}}(\mathrm{m})$ there is a canonical $S L_{2}$ isomorphism $\psi: W^{p} \rightarrow A^{p}$ satisfying the property: for any $\sigma \in \Sigma$ and $p^{\prime}, p^{\prime \prime} \in \Lambda_{\mathcal{B}}(\sigma)$ such that $p=$ $p^{\prime}+p^{\prime \prime}$, diagram (18) commutes. Set $\phi_{p}=\psi$ and define $\mathcal{F}_{\mathfrak{m}}=\left\{\phi_{p}: W^{p} \rightarrow A^{p}\right\}_{p \in \Lambda_{\mathcal{B}}(m)}$. This completes the induction.

Let $\mathcal{F}=\bigcup_{\mathfrak{m}=1}^{\infty} \mathcal{F}_{\mathrm{m}}$. By construction, $\mathcal{F}$ is a compatible family of $S L_{2}$-isomorphisms. This completes the proof of the first statement of the proposition.

Now suppose $\widetilde{\mathcal{F}}=\left\{\widetilde{\phi}_{\mathrm{p}}: W^{\mathfrak{p}} \rightarrow A^{\mathfrak{p}}\right\}_{\mathfrak{p} \in \Lambda_{\mathcal{B}}}$ is another compatible family of $\mathrm{SL}_{2}$-isomorphisms. We will show by induction on $p_{\max }$ that there exist a set of nonzero scalars $\left\{c_{p} \in \mathbb{C}^{\times}\right.$: $\left.p \in \Lambda_{\mathcal{B}}\right\}$, such that $\phi_{p}=c_{p} \widetilde{\phi}_{p}$ for all $p \in \Lambda_{\mathcal{B}}$.

We already noted that by Schur's Lemma each isomorphism $\phi_{\mathrm{p}}$ with $p_{\max }=1$ is unique up to scalar. Therefore there exist $c_{p} \in \mathbb{C}^{\times}$such that

$$
\phi_{p}=c_{p} \widetilde{\phi}_{p}
$$

for all $p$ with $p_{\max }=1$. Let $m>1$. Suppose now that there exist scalars so that (21) holds for all $p \in \Lambda_{\mathcal{B}}$ such that $p_{\max }<m$. Let $p \in \Lambda_{\mathcal{B}}$ with $p_{\max }=m$. Choose some $\sigma \in \Sigma$ such that $p \in \Lambda_{\mathcal{B}}(\sigma)$. By Lemma 6.3, there exist $p^{\prime}, p^{\prime \prime} \in \Lambda_{\mathcal{B}}(\sigma, m-1)$ such that 
$p=p^{\prime}+p^{\prime \prime}$. Then by the compatibility of $\mathcal{F}$ the following diagram commutes:

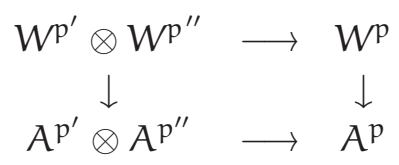

where the vertical maps are $\phi_{p^{\prime}} \otimes \phi_{p^{\prime \prime}}$ and $\phi_{p}$. By hypothesis,

$$
\phi_{\mathfrak{p}^{\prime}} \otimes \phi_{\mathfrak{p}^{\prime \prime}}=c_{\mathfrak{p}^{\prime}} c_{\mathrm{p}^{\prime \prime}} \widetilde{\phi}_{\mathrm{p}^{\prime}} \otimes \widetilde{\phi}_{\mathrm{p}^{\prime \prime}}
$$

Therefore (22) commutes with the vertical maps replaced by $\widetilde{\phi}_{\mathfrak{p}^{\prime}} \otimes \widetilde{\phi}_{\mathrm{p}^{\prime \prime}}$ and $\frac{1}{c_{p^{\prime}} c_{p^{\prime \prime}}} \phi_{p}$. Hence $\frac{1}{c_{p^{\prime}} c_{p^{\prime \prime}}} \phi_{p}=\widetilde{\phi}_{p}$, or, in other words, $c_{p}=c_{p^{\prime}} c_{p^{\prime \prime}}$ and $\phi_{p}=c_{p} \widetilde{\phi}_{p}$. This completes the induction, and shows that $\phi_{p} \in \mathcal{F}$ is unique up to scalar.

We can now prove Proposition 3.7. Indeed, let $\mathcal{F}=\left\{\phi_{p}: W^{p} \rightarrow A^{p}\right\}_{p \in \wedge_{\mathcal{B}}}$ be a compatible family of $\mathrm{SL}_{2}$-isomorphisms guaranteed by the above proposition. Define a map

$$
\phi_{\sigma}: \mathcal{B}_{\sigma} \rightarrow \mathcal{A}_{\sigma}
$$

by

$$
\left.\phi_{\sigma}\right|_{W^{p}}=\phi_{p}
$$

for all $p \in \Lambda_{\mathcal{B}}(\sigma)$, and extend linearly. Since $\mathcal{F}$ is a compatible family, $\phi_{\sigma}$ is an isomorphism of $S_{2}$-algebras. Indeed, the commutativity of diagram (16) means precisely that $\phi_{\sigma}$ is an algebra homomorphism.

Now suppose $\widetilde{\phi}_{\sigma}: \mathcal{B}_{\sigma} \rightarrow \mathcal{A}_{\sigma}$ is some other isomorphism of $\mathrm{SL}_{2}$-algebras. Set $\widetilde{\phi}_{\mathrm{p}}=$ $\left.\widetilde{\phi}_{\sigma}\right|_{W^{p}}$. Then

$$
\left\{\widetilde{\phi}_{p}: p \in \Lambda_{\mathcal{B}}(\sigma)\right\}
$$

is a compatible family of $\mathrm{SL}_{2}$ isomorphisms. Therefore, by the above proposition, there scalars $c_{p}$ such that $\phi_{p}=c_{p} \widetilde{\phi}_{p}$. Therefore the graded components of $\widetilde{\phi}_{\sigma}$ are scalar multiples of the graded components of $\phi_{\sigma}$, i.e. $\phi_{\sigma}$ is unique up to scalars. This proves part (1) of Proposition 3.7

To prove part (2) we define a representation of $L$ on $\mathcal{B}_{\sigma}$, denoted $\Phi_{\sigma}$, by the formula

$$
\Phi_{\sigma}(g)=\phi_{\sigma}^{-1} \circ \theta_{\sigma}(g) \circ \phi_{\sigma} .
$$

Here $\mathrm{g} \in \mathrm{L}, \phi_{\sigma}$ is the algebra isomorphism coming from Proposition 6.9 as in (23), and $\theta_{\sigma}$ is the action of $\mathrm{L}$ on $\mathcal{A}_{\sigma}$ defined in (8). We must show that $\Phi_{\sigma}$ is independent of the choice of $\phi_{\sigma}$.

Indeed, suppose we are given another isomorphism $\widetilde{\phi}_{\sigma}$ and use it to define the corresponding representation of $L$ on $\mathcal{B}_{\sigma}$, which we denote $\widetilde{\Phi}_{\sigma}$. Now, choose scalars $c_{p}$ as above. Then for any $g \in \mathrm{L}$ and $p \in \Lambda_{\mathcal{B}}(\sigma)$,

$$
\begin{aligned}
\left.\Phi_{\sigma}(g)\right|_{W^{p}} & =\phi_{p}^{-1} \circ \theta_{p}(g) \circ \phi_{p} \\
& =\left(c_{p} \widetilde{\phi}_{p}\right)^{-1} \circ \theta_{p}(g) \circ\left(c_{p} \widetilde{\phi}_{p}\right) \\
& =\left(\widetilde{\phi}_{p}\right)^{-1} \circ \theta_{p}(g) \circ \widetilde{\phi}_{p} \\
& =\left.\widetilde{\Phi}_{\sigma}(g)\right|_{W^{p}} .
\end{aligned}
$$


Therefore $\Phi_{\sigma}=\widetilde{\Phi}_{\sigma}$. This proves part (2) of Proposition 3.7 .

6.3. Proof of Theorem 3.5. Existence: Consider the representations $\left(\Phi_{\sigma}, \mathcal{B}_{\sigma}\right)$ of $\mathrm{L}$ from Proposition 3.7, and let mathcalF be the compatible family guaranteed by Proposition 6.9. These representations satisfy four desirable properties, all of which are almost tautologies.

(i) For any $p \in \Lambda_{\mathcal{B}}(\sigma), W^{p}$ is an irreducible L-submodule isomorphic to $\bigotimes_{i=1}^{n} F^{r_{i}}(p)$. Indeed, by definition of $\Phi_{\sigma}, \phi_{p}: W^{p} \rightarrow A^{p}$ is an isomorphism of L-modules.

(ii) $\mathrm{L}$ acts as algebra automorphisms on $\mathcal{B}_{\sigma}$. In other words, we claim that for $p, p^{\prime} \in$ $\Lambda_{\mathcal{B}}(\sigma)$, the product map, $W^{p} \otimes W^{p^{\prime}} \rightarrow W^{p+p^{\prime}}$, is a homomorphism of L-modules. Indeed, by the compatibility of $\mathcal{F}$, the product map factors as follows:

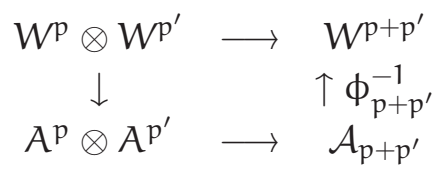

Since the three lower maps are L-module morphisms, it follows that the top map is too.

(iii) $\operatorname{Res}_{S L_{2}}^{\mathrm{L}}\left(\Phi_{\sigma}\right)$ is the natural action of $S L_{2}$ on $\mathcal{B}_{\sigma}$. In other words, for $x \in S L_{2}$

$$
\left.\chi\right|_{\mathcal{B}_{\sigma}}=\Phi_{\sigma}(\delta(x))
$$

where $\left.x\right|_{\mathcal{B}_{\sigma}}$ denotes the natural action of $x$ on $\mathcal{B}_{\sigma}$ and $\delta$ is the diagonal embedding of $\mathrm{SL}_{2}$ into L. Indeed, $\phi_{\sigma}$ intertwines the natural action of $\mathrm{SL}_{2}$ on $\mathcal{B}_{\sigma}$ with the diagonal $\mathrm{SL}_{2}$-action on $\mathcal{A}_{\sigma}$. This means that

$$
\phi_{\sigma} \circ\left(\left.x\right|_{\mathcal{B}_{\sigma}}\right)=\theta_{\sigma}(\delta(x)) \circ \phi_{\sigma}
$$

Therefore

$$
\begin{aligned}
\Phi_{\sigma}(\delta(x)) & =\phi_{\sigma}^{-1} \circ \theta_{\sigma}(\delta(x)) \circ \phi_{\sigma} \\
& =\phi_{\sigma}^{-1} \circ \phi_{\sigma} \circ\left(\left.x\right|_{\mathcal{B}_{\sigma}}\right) \\
& =\left.x\right|_{\mathcal{B}_{\sigma}} .
\end{aligned}
$$

(iv) For any $\sigma_{1}, \sigma_{2} \in \Sigma$ and $g \in \mathrm{L}$

$$
\left.\Phi_{\sigma_{1}}(\mathrm{~g})\right|_{\mathcal{B}_{\sigma_{1}} \cap \mathcal{B}_{\sigma_{2}}}=\left.\Phi_{\sigma_{2}}(\mathrm{~g})\right|_{\mathcal{B}_{\sigma_{1}} \cap \mathcal{B}_{\sigma_{2}}}
$$

Indeed, suppose $p \in \Lambda_{\mathcal{B}}\left(\sigma_{1}\right) \cap \Lambda_{\mathcal{B}}\left(\sigma_{2}\right)$. Then $\left.\phi_{\sigma_{1}}\right|_{W^{p}}=\phi_{p}=\left.\phi_{\sigma_{2}}\right|_{W^{p}}$ from which (24) immediately follows.

We now construct from $\mathcal{F}$ the representation $\left(\Phi_{\mathcal{F}}, \mathcal{B}\right)$ of $\mathrm{L}$ satisfying the conditions of Theorem 3.5. Let $g \in \mathrm{L}$. Define $\Phi_{\mathcal{F}}(\mathrm{g})$ on $\mathcal{B}$ by

$$
\left.\Phi_{\mathcal{F}}(g)\right|_{\mathcal{B}_{\sigma}}=\Phi_{\sigma}(g) \text {. }
$$

By (24) this is well-defined, and since $\sum_{\sigma} \mathcal{B}_{\sigma}=\mathcal{B}$, this gives an action of $\mathrm{L}$ on all of $\mathcal{B}$. Moreover, by properties (i) and (ii), $\left(\Phi_{\mathcal{F}}, \mathcal{B}\right)$ satisfies the conditions of Theorem 3.5. By property (iii) $\left(\Phi_{\mathcal{F}}, \mathcal{B}\right)$ extends the natural action of $S L_{2}$ on $\mathcal{B}$.

Uniqueness: Suppose $(\Phi, \mathcal{B})$ is some representation of $\mathrm{L}$ satisfying the conditions of Theorem 3.5, By Proposition 3.7, to show uniqueness it suffices to show that there exists a compatible family $\widetilde{\mathcal{F}}=\left\{\widetilde{\phi}_{\mathfrak{p}}: W^{p} \rightarrow A^{p}\right\}_{\mathfrak{p} \in \Lambda_{\mathcal{B}}}$ such that $\Phi=\Phi_{\widetilde{\mathcal{F}}}$. 
By condition (1) of the theorem, $W^{p}$ is isomorphic to $A^{p}$ as L-modules for every $p \in \Lambda_{\mathcal{B}}$. In particular, we can choose a set of L-isomorphisms $\left\{\widetilde{\phi}_{p}: W^{p} \rightarrow A^{p}\right\}_{p_{\max }=1}$. From this set we construct a compatible family $\widetilde{\mathcal{F}}=\left\{\widetilde{\phi}_{p}: W^{p} \rightarrow A^{p}\right\}_{p} \in \Lambda_{\mathcal{B}}$ as in the proof of Proposition 6.9.

To show that $\Phi=\Phi_{\widetilde{\mathcal{F}}}$, we need to show that for all $g \in \mathrm{L}$ and $p \in \Lambda_{\mathcal{B}}$, the following diagram commutes:

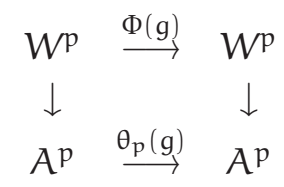

where the vertical maps are both $\widetilde{\phi}_{\mathrm{p}}$. We prove this by induction on $p_{\max }$.

Let $p \in \Lambda_{\mathcal{B}}$. If $p_{\max }=1$, then (25) commutes by our choice of $\left\{\widetilde{\phi}_{p}: W^{p} \rightarrow A^{p}\right\}_{p_{\max }=1}$ above. Let $m>1$ and assume (25) commutes for all $p$ such that $p_{\max }<m$. Suppose then that $p_{\max }=m$. Choose some $\sigma \in \Sigma$ such that $p \in \Lambda_{\mathcal{B}}(\sigma)$. By Lemma 6.3, there exist $p^{\prime}, p^{\prime \prime} \in \Lambda_{\mathcal{B}}(\sigma, m-1)$ such that $p=p^{\prime}+p^{\prime \prime}$. Consider the following cube:

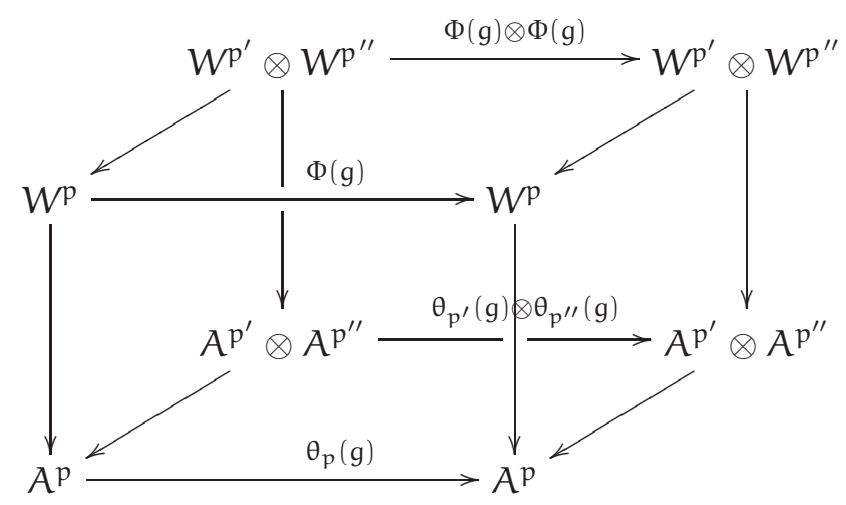

The top square commutes since $\Phi$ satisfies condition (2) of Theorem 3.5. The left and right squares commute by the compatibility of $\mathcal{F}$. The bottom square commutes since the product on $\mathcal{A}_{\sigma}$ intertwines the L-action. Finally, the back square commutes by inductive hypothesis. Since all the maps are surjective, we conclude that the front square commutes. This completes the induction, and proves that $\Phi=\Phi_{\tilde{\mathcal{F}}}$.

This completes the proof of Theorem 3.5 .

\section{Proof of Corollary 3.8}

Let $T_{S L_{2}}$ be the torus of $S L_{2}$ consisting of diagonal matrices. Let $T_{L}=T_{S L_{2}} \times \cdots \times T_{S L_{2}}$ be the diagonal torus of L. From elementary representation theory of $\mathrm{SL}_{2}$ we know that irreducible L-modules decompose canonically into one dimensional $\mathrm{T}_{\mathrm{L}}$ weight spaces. Now let $(\Phi, \mathcal{B})$ be the representation of $\mathrm{L}$ afforded by Theorem 3.5. Then by part (1) of Theorem 3.5, the multiplicity spaces $W^{p},\left(p \in \Lambda_{\mathcal{B}}\right)$, decompose into one dimensional spaces. 
The decomposition is canonical. Indeed, $T_{L}$ is the unique torus of $L$ containing $T_{S L_{2}}$. Moreover, the choice of the torus $\mathrm{T}_{\mathrm{SL}_{2}}$ is induced by our choice of torus of $S p_{n}$, i.e.

$$
\mathrm{T}_{\mathrm{SL}_{2}}=\mathrm{SL}_{2} \cap \mathrm{T}_{\mathrm{C}_{\mathrm{n}}} \text {. }
$$

Therefore the decomposition of $W^{\lambda / \mu}$ into one dimensional spaces depends only the choice of torus of $S p_{n}$. We now make this decomposition more precise.

Lemma 7.1. Let $\lambda / \mu \in \Lambda_{\mathcal{B}}$. The weight spaces of $\mathrm{T}_{\mathrm{L}}$ on $\mathrm{W}^{\lambda / \mu}$ are indexed by the set $\left\{\gamma \in \Lambda_{n}: \mu<\gamma<\lambda^{+}\right\}$. An element $\gamma$ corresponds to the weight

$$
\left(t_{1}, \ldots, t_{n}\right) \mapsto \prod_{i=1}^{n} t_{i}^{2 \gamma_{i}-x_{i}-y_{i}} .
$$

where $h(\lambda / \mu)=\left(x_{1}, y_{1}, \ldots, x_{n}, y_{n}\right)$.

Proof. Set $p=\lambda / \mu$. Since $W^{p}$ is isomorphic as an L-module to $\bigotimes_{i=1}^{n} F^{r_{i}(p)}$, the weight spaces of $T_{L}$ on $W^{p}$ are indexed by

$$
\left\{\left(j_{1}, \ldots, j_{n}\right): 0 \leq j_{i} \leq r_{i}(p) \text { for } i=1, \ldots, n\right\} .
$$

Indeed, such a sequence $\left(j_{1}, \ldots, j_{n}\right)$ corresponds to the weight

$$
\left(t_{1}, \ldots, t_{n}\right) \mapsto \prod_{i=1}^{n} t_{i}^{\left(-r_{i}(p)+2 j_{i}\right)} .
$$

Now there is a one-to-one correspondence between

$$
\left\{\left(j_{1}, \ldots, j_{n}\right): 0 \leq j_{i} \leq r_{i}(p) \text { for } i=1, \ldots, n\right\}
$$

and

$$
\left\{\left(\gamma_{1}, \ldots, \gamma_{n}\right): y_{i} \leq \gamma_{i} \leq x_{i} \text { for } i=1, \ldots, n\right\}
$$

given by $\left(j_{1}, \ldots, j_{n}\right) \mapsto\left(j_{1}+y_{1}, \ldots, j_{n}+y_{n}\right)$. Therefore, by Lemma 5.1, the weight spaces of $\mathrm{T}_{\mathrm{L}}$ on $\mathrm{W}^{\mathrm{p}}$ are indexed by $\left\{\gamma \in \Lambda_{n}: \mu<\gamma<\lambda^{+}\right\}$. Unwinding these identifications, we see that a pattern $\gamma$ corresponds to the weight

$$
\left(t_{1}, \ldots, t_{n}\right) \mapsto \prod_{i=1}^{n} t_{i}^{-\left(x_{i}+y_{i}\right)+2 \gamma_{i}}
$$

For $\lambda / \mu \in \Lambda_{\mathcal{B}}$ and $\gamma \in\left\{\gamma \in \Lambda_{n}: \mu<\gamma<\lambda^{+}\right\}$, let $W^{\lambda / \gamma / \mu}$ be the $T_{L}$ weight space of $W^{\lambda / \mu}$ corresponding to the weight $\gamma$ by the above lemma. Then we obtain a decomposition of $W^{\lambda / \mu}$ into one dimensional weight spaces:

$$
W^{\lambda / \mu}=\bigoplus_{\substack{\gamma \in \Lambda_{n} \\ \mu<\gamma<\lambda^{+}}} W^{\lambda / \gamma / \mu} .
$$

This decomposition is canonical in the sense that it only depends on the choice of torus $\mathrm{T}_{\mathrm{C}_{\mathrm{n}}} \subset S \mathrm{p}_{2 \mathrm{n}}$. This completes the proof of Corollary 3.8. 


\section{REFERENCES}

[GZ50] Gel'fand, I. M.; Zetlin, M. L. Finite-dimensional representations of the group of unimodular matrices. (Russian) Doklady Akad. Nauk SSSR (N.S.) 71, (1950). 825-828.

[GW09] Goodman, Roe; Wallach, Nolan R. Symmetry, Representations, and Invariants. Graduate Texts in Mathematics, 255. Springer, Dodrecht, 2009.

[HTW08] Howe, Roger; Tan, Eng-Chye; Willenbring, Jeb Reciprocity Algebras and Branching for Classical Symmetric Pairs, Groups and Analysis - the Legacy of Hermann Weyl, London Mathematical Society Lecture Notes, 354, Cambridge University Press, 2008.

[KY10] Kim, Sangjib; Yacobi, Oded, A basis for the symplectic group branching algebra. Preprint 2010.

[Mol99] Molev, A. I. A basis for representations of symplectic Lie algebras. Comm. Math. Phys. 201 (1999), no. 3, 591-618.

[Vin95] Vinberg, Ernest B. The asymptotic semigroup of a semisimple Lie group. Semigroups in algebra, geometry and analysis (Oberwolfach, 1993), 293-310, de Gruyter Exp. Math., 20, de Gruyter, Berlin, 1995.

[WY] Wallach, Nolan; Yacobi, Oded, A multiplicity formula for tensor products of $\mathrm{SL}_{2}$ modules and an explicit $S p_{2 n}$ to $S p_{2 n-2} \times S p_{2}$ branching formula, Contemp. Math., American Mathematical Society, Providence, R.I., 2009, (to appear).

[Zh62] Zhelobenko, D.P. The classical groups. Spectral analysis of their finite dimensional representations, Russ. Math. Surv. 17 (1962), 1-94.

[Zh73] Zhelobenko, D. P. Compact Lie groups and their representations. Translated from the Russian by Israel Program for Scientific Translations. Translations of Mathematical Monographs, Vol. 40. American Mathematical Society, Providence, R.I., 1973.

E-mail address: oyacobi@math.tau.ac.il

School of Mathematical Sciences, Tel Aviv University, Tel Aviv 69978, Israel 